\title{
SPECTRAL MEASURES AND CUNTZ ALGEBRAS
}

\author{
DORIN ERVIN DUTKAY AND PALLE E. T. JORGENSEN
}

\begin{abstract}
We consider a family of measures $\mu$ supported in $\mathbb{R}^{d}$ and generated in the sense of Hutchinson by a finite family of affine transformations. It is known that interesting sub-families of these measures allow for an orthogonal basis in $L^{2}(\mu)$ consisting of complex exponentials, i.e., a Fourier basis corresponding to a discrete subset $\Gamma$ in $\mathbb{R}^{d}$. Here we offer two computational devices for understanding the interplay between the possibilities for such sets $\Gamma$ (spectrum) and the measures $\mu$ themselves. Our computations combine the following three tools: duality, discrete harmonic analysis, and dynamical systems based on representations of the Cuntz $C^{*}$-algebras $\mathcal{O}_{N}$.
\end{abstract}

\section{INTRODUCTION}

The idea of self-similarity is ubiquitous in pure and applied mathematics and has many incarnations, and even more applications. In this generality the method often goes by the name multi-scale theory; see for example [ZHSS09. We are faced with a fixed system $S$, say finite, of transformations. The mappings from $S$ are applied to the data at hand, and we look for similarities. A single measure, say $\mu$, may be transformed with the mappings in $S$. If $\mu$ is a convex combination of the resulting measures, we say that $\mu$ is a self-similar measure. Here we aim for a harmonic analysis of the Hilbert space $L^{2}(\mu)$ in the event $\mu$ is selfsimilar. However, this is meaningful only if further restrictions are placed on the setup. For example, we will assume that $S$ consists of affine and contractive transformations in $\mathbb{R}^{d}$ for some fixed dimension $d$; and we will assume that the affine transformations arise from scaling with the same matrix for the different mappings in $S$. In this case $\mu$ is the result of an iteration in the small, and its support is a compact subset in $\mathbb{R}^{d}$. We explore algorithms for generating Fourier bases of complex exponentials in $L^{2}(\mu)$ by iteration in the large.

A word about the Cuntz algebras $\mathcal{O}_{N}$ from the title of our paper: These are infinite algebras on a finite number of generators, the following relations

$$
S_{i}^{*} S_{j}=\delta_{i j} 1, \quad(i, j \in\{1, \ldots, N\}), \quad \sum_{i=1}^{N} S_{i} S_{i}^{*}=1 .
$$

They are intrinsically selfsimilar and therefore ideally serve to encode iterated function systems (IFSs) from geometric analysis, and their measures $\mu$. At the same time, their representations offer (in a more subtle way) a new harmonic analysis of the associated $L^{2}(\mu)$-Hilbert spaces. Even though the Cuntz algebras initially

Received by the editor January 25, 2010 and, in revised form, July 14, 2011.

2010 Mathematics Subject Classification. Primary 28A80, 42B05, 46C05, 46L89.

Key words and phrases. Spectrum, Hilbert space, fractal, Fourier bases, selfsimilar, iterated function system, operator algebras.

With partial support by the National Science Foundation. 
entered into the study of $C^{*}$-algebras Cun77] and physics, in recent years these same Cuntz algebras, and their representation, have found increasing use in both pure and applied problems, wavelets, fractals, signals; see for example [Jor06.

In our paper we address simultaneously problems in spectral geometry and in representations of Cuntz relations $\mathcal{O}_{N}$. These connections prove to be fundamental in a number of ways. More specifically, we are concerned with links between, on the one hand, (i) fundamental problems for affine fractals and their spectral theory, and (ii) representations of the Cuntz relations on the other. We show that, indeed, these links capture essential features from both areas. We further show that key results from one have important implications for the other. Thus, our theorems serve to emphasize advances at the cross roads of the two.

To motivate this, we note the following aspects characterizing the two settings:

Fractals and spectra: (i) Inherent in the study of affine fractals, both the measures and their support, is a geometric notion of affine self-similarity. This refers to self-similarity in the small. In the study of Fourier duality, one aims for a Fourier spectrum built from a recursive scaling law that generates spectrum from an expanding (and dual) notion of self-similarity, so affine self-similarity transformations; these are then spectra realized as "fractals in the large".

We show that key notions from (i) are captured very naturally in (ii); and we explore the implications.

Since spectral theory takes place in Hilbert spaces $L^{2}(\mu)$ for affine self-similar measures, the introduction of representations of the Cuntz relations allows us to transfer spectral duality questions to a framework in geometry of Hilbert space, which in turn is ideally suited for questions from (i). So the translation between (i) and geometry for systems of isometries in Hilbert space; see (1.1) above.

This allows us to use (ii), representation theory for isometries in Hilbert space, in order to identify new self-similar spectral notions from (i).

Conversely, our solutions to problems from (i), (Corollary 3.7, Theorem 4.6] and Corollary 4.21) produce unexpected representations of the Cuntz relations going beyond those studied earlier; e.g., BJ97a, BJ97b, BJ99. This is of significance in representation theory as it is known that each Cuntz algebra $\mathcal{O}_{N}$ is purely infinite; and, even further, that the equivalence classes of its irreducible representations do not admit a "Borel cross section", i.e., a labeling with an isomorphism in a Borel category; see e.g., BJKW00.

Earlier work along these lines include the papers DJ06a, DJ07, Fug74, HL08a, Jør82, Ped04, Tao04; as well as an array of diverse applications ACHM07, BV05, CM07, DLC09, HL08b, MZ09, OS05, YALC03. Recent papers by Barnsley et al. Bar09, BHS05 show that even the restricted family of affine IFSs suffices for such varied applications as vision, music, Gabor transform processing, and Monte Carlo algorithms. The literature is large and we direct the reader to [DJ07 for additional references. Our present study suggests algorithms for this problem based on representations of a certain scale of $C^{*}$-algebras (the Cuntz algebras Cun77]); see also Jor06. In addition, we shall make use of Hadamard matrices AdLM02, CHK97, Dit04, GR09, KS93]; number theory and tilings [BL07, FLS09, LL07, MP04]; and of tools from symbolic dynamics [Den09, Tho05, DFdGtHR04, MM09.

Our starting point is a system $(R, B, L)$ in $\mathbb{R}^{d}$ subject to the conditions from Definitions 1.3. i.e., a Hadamard triple. With the given expansive matrix $R$ and the vectors from the finite set $B$ we build one affine iterated function system (IFS) 
(1.2), and the associated Hutchinson measure $\mu_{B}$. Our objective is to set up an algorithmic approach for constructing orthogonal families of complex exponentials in $L^{2}\left(\mu_{B}\right)$, so-called Fourier bases.

With the third part of the triple $(R, B, L)$, the finite set of vectors from $L$ and the transposed matrix $R^{T}$ we build a second IFS (1.13), the $L$-system, now with $R^{T}$ as scaling matrix and the vectors from $L$ as translations. The set $L$, together with the transpose matrix $R^{T}$ will be used to construct the frequencies associated to the Fourier basis. Due to the Hadamard property which is assumed for the combined system $(R, B, L)$, we then get a natural representation of the Cuntz algebra $\mathcal{O}_{N}$ (where $N=\# L=\# B$ ) with the generating operators $\left\{S_{l}: l \in L\right\}$ acting as isometries in $L^{2}\left(\mu_{B}\right)$; see (1.10) in Proposition 1.4

Our objective is to use this Cuntz algebra representation in order to recursively construct and exhibit an orthogonal family of complex exponentials in $L^{2}\left(\mu_{B}\right)$. For this purpose we introduce a family of minimal and finite cycles $C$ for the $L$-system, which are extreme in the sense made precise in Definition 3.1. For each of the extreme cycles $C$, with the $L$-Cuntz representation, we then generate an infinite orthogonal family $\Gamma(C)$ of complex exponentials in $L^{2}\left(\mu_{B}\right)$. With the cycle $C$ fixed, we prove that the corresponding closed subspace $\mathcal{H}(C)$ now reduces the Cuntzalgebra representation, and further that the restricted representation is irreducible. For distinct minimal cycles we get orthogonal subspaces $\mathcal{H}(C)$ and corresponding disjoint representations (see Theorem 3.4).

A key tool in the study of these representations of the Cuntz algebra is a transfer operator $R_{B, L}$ (Definition 1.7), analogous to a transfer operator used first by David Ruelle in a different context. In Corollary 1.13 we show that the reduction of the $L$-Cuntz representation (1.10) is accounted for by a family of harmonic functions $h_{C}$ for the transfer operator $R_{B, L}$.

Finally, we prove that the sum of the functions $h_{C}$ equals to the constant function 1 if and only if the union of the sets $\Gamma(C)$ forms an orthogonal basis of Fourier exponentials in $L^{2}\left(\mu_{B}\right)$.

In section 1 , we define the representation of the Cuntz algebra $\left(S_{l}\right)_{l \in L}$ associated to a Hadamard pair and we present some computational features of this representation. We show in Proposition 1.12 and Corollary 1.13 how the canonical endomorphism on $\mathcal{B}\left(L^{2}\left(\mu_{B}\right)\right)$, constructed from the representation:

$$
\alpha(T)=\sum_{l \in L} S_{l} T S_{l}^{*}, \quad\left(T \in \mathcal{B}\left(L^{2}\left(\mu_{B}\right)\right)\right)
$$

is connected to the transfer operator associated to the "dual" IFS $\left(\tau_{l}\right)_{l \in L}$.

In section 2 we revisit the permutative representations defined in [BJ99]. They are needed for the decomposition of our representation $\left(S_{l}\right)_{l \in L}$ into irreducible representations. We will see in section 3, that extreme cycles (Definition 3.1) will generate irreducible atoms in this decomposition (Theorem 3.4). In dimension 1, we know from [DJ06b] that the extreme cycles offer a complete description of the picture. However, in higher dimensions, more complicated decompositions might appear. We analyze these possibilities in section 4, and we illustrate it in Example 4.7.

Definition 1.1. We will denote by $e_{t}$ the exponential function

$$
e_{t}(x)=e^{2 \pi i t \cdot x}, \quad\left(x, t \in \mathbb{R}^{d}\right)
$$


Let $\mu$ be a Borel probability measure on $\mathbb{R}^{d}$. We say that $\mu$ is a spectral measure if there exists a subset $\Lambda$ of $\mathbb{R}^{d}$ such that the family $E(\Lambda):=\left\{e_{\lambda}: \lambda \in \Lambda\right\}$ is an orthonormal basis for $L^{2}(\mu)$. In this case $\Lambda$ is called a spectrum for the measure $\mu$ and we say that $(\mu, \Lambda)$ is aspectral pair.

Definition 1.2. We will use the following assumptions throughout the paper. Let $R$ be a $d \times d$ expansive integer matrix, i.e., all its eigenvalues have absolute value strictly bigger than one, and let $B$ be a finite subset of $\mathbb{Z}^{d}$, with $0 \in B$. We denote by $N$ the cardinality of $B$. Define the maps

$$
\tau_{b}(x)=R^{-1}(x+b), \quad\left(x \in \mathbb{R}^{d}, b \in B\right) .
$$
$B$.

We call $\left(\tau_{b}\right)_{b \in B}$ the (affine) iterated function system (IFS) associated to $R$ and

By [Hut81, there exists a unique compact set $X_{B}$ called the attractor of the IFS $\left(\tau_{b}\right)_{b \in B}$ such that

$$
X_{B}=\bigcup_{b \in B} \tau_{b}\left(X_{B}\right)
$$

In our case, it can be written explicitly

$$
X_{B}=\left\{\sum_{k=1}^{\infty} R^{-k} b_{k}: b_{k} \in B \text { for all } k \geq 1\right\} .
$$

We will use also the notation $X(B)$ for $X_{B}$.

There exists a unique Borel probability measure $\mu_{B}$ such that

$$
\mu_{B}(E)=\frac{1}{N} \sum_{b \in B} \mu_{B}\left(\tau_{b}^{-1}(E)\right) \text { for all Borel subsets } E \text { of } \mathbb{R}^{d} .
$$

Equivalently,

$$
\int f d \mu_{B}=\frac{1}{N} \sum_{b \in B} \int f \circ \tau_{b} d \mu_{B} \text { for all bounded Borel function } f \text { on } \mathbb{R}^{d} \text {. }
$$

The measure $\mu_{B}$ is called the invariant measure of the IFS $\left(\tau_{b}\right)_{b \in B}$. It is supported on $X_{B}$.

We say that the measure $\mu_{B}$ has no overlap if

$$
\mu_{B}\left(\tau_{b}\left(X_{B}\right) \cap \tau_{b^{\prime}}\left(X_{B}\right)\right)=0, \text { for all } b \neq b^{\prime} \in B .
$$

If the measure $\mu_{B}$ has no overlap, then one can define the map $\mathcal{R}: X_{B} \rightarrow X_{B}$

$$
\mathcal{R}(x)=R x-b, \text { if } x \in \tau_{b}\left(X_{B}\right) .
$$

The map $\mathcal{R}$ is well defined $\mu_{B}$-a.e. on $X_{B}$.

Definition 1.3. Let $R$ be a $d \times d$ integer matrix, and $B$ and $L$ two subsets of $\mathbb{Z}^{d}$ of the same cardinality $N$, with $0 \in B$ and $0 \in L$. We say that $(B, L)$ forms a Hadamard pair if the following matrix is unitary:

$$
\frac{1}{\sqrt{N}}\left(e^{2 \pi i R^{-1} b \cdot l}\right)_{b \in B, l \in L} .
$$

Throughout, the Hilbert space considered is $L^{2}\left(\mu_{B}\right)$ unless otherwise specified, and we introduce specific families of operators acting there, starting with the operator system $\left(S_{l}\right)_{l \in L}$ in (1.10) below. This system $\left(S_{l}\right)_{l \in L}$ will be fixed, and it defines a representation of the Cuntz algebra $\mathcal{O}_{N}$ with $N=\# L$. 
Proposition 1.4. Let $\left(\tau_{b}\right)_{b \in B}$ be as in Definition 1.2 and assume the invariant measure $\mu_{B}$ has no overlap. Let $L$ be a subset of $\mathbb{Z}^{d}$ with the same cardinality $N$ as $B$. Define the operators on $L^{2}\left(\mu_{B}\right)$ :

$$
\left(S_{l} f\right)(x)=e^{2 \pi i l \cdot x} f(\mathcal{R} x), \quad\left(x \in X_{B}, f \in L^{2}\left(\mu_{B}\right)\right)
$$

(i) The operator $S_{l}$ is an isometry for all $l \in L$.

(ii) The operators $\left(S_{l}\right)_{l \in L}$ form a representation of the Cuntz algebra $\mathcal{O}_{N}$ if and only if $(B, L)$ forms a Hadamard pair.

Proof. We will need the following lemma:

Lemma 1.5. If the measure $\mu_{B}$ has no overlap then for all $b \in B$ and all integrable Borel functions $f$ :

$$
\int_{\tau_{b}\left(X_{B}\right)} f d \mu_{B}=\frac{1}{N} \int f \circ \tau_{b} d \mu_{B}
$$

Proof. From the invariance equation

$$
N \int \chi_{\tau_{b}\left(X_{B}\right)} g d \mu_{B}=\sum_{b^{\prime} \in B} \int \chi_{\tau_{b}\left(X_{B}\right)} \circ \tau_{b}^{\prime} g \circ \tau_{b}^{\prime} d \mu_{B} .
$$

Since there is no overlap $\chi_{\tau_{b}\left(X_{B}\right)} \circ \tau_{b^{\prime}}$ is 1 if $b=b^{\prime}$, and is 0 if $b \neq b^{\prime}, \mu_{B^{-a l m o s t}}$ everywhere (since $\mu_{B}$ is supported on $X_{B}$ ).

This leads to the conclusion.

We prove (i). Take $f \in L^{2}\left(\mu_{B}\right), l \in L$. Then, with Lemma 1.5]

$$
\begin{gathered}
\int\left|e^{2 \pi i l \cdot x} f(\mathcal{R} x)\right|^{2} d \mu_{B}=\sum_{b \in B} \int_{\tau_{b}\left(X_{B}\right)}|f(R x-b)|^{2} d \mu_{B}(x) \\
=\sum_{b \in B} \frac{1}{N} \int\left|f\left(R \tau_{b}(x)-b\right)\right|^{2} d \mu_{B}(x)=\int|f|^{2} d \mu_{B} .
\end{gathered}
$$

This shows that $S_{l}$ is an isometry.

We now prove (ii). For this, we have to compute $S_{l}^{*}$. We have, for $f, g \in L^{2}\left(\mu_{B}\right)$ :

$$
\begin{aligned}
\left\langle S_{l} f, g\right\rangle & =\int e^{2 \pi i l \cdot x} f(\mathcal{R} x) \bar{g}(x) d \mu_{B}(x) \\
& =\sum_{b \in B} \int_{\tau_{b}(X)} e^{2 \pi i \cdot x} f(R x-b) \bar{g}(x) d \mu_{B}(x), \text { and with Lemma 1.5 } \\
& =\frac{1}{N} \sum_{b \in B} \int e^{2 \pi i \cdot \tau_{b}(x)} f(x) \bar{g}\left(\tau_{b}(x)\right) d \mu_{B}(x) .
\end{aligned}
$$

This shows that

$$
\left(S_{l}^{*} g\right)(x)=\frac{1}{N} \sum_{b \in B} e^{-2 \pi i l \cdot \tau_{b}(x)} g\left(\tau_{b}(x)\right), \quad\left(x \in X_{B}, g \in L^{2}\left(\mu_{B}\right)\right) .
$$

Then

$$
\begin{aligned}
S_{l}^{*} S_{l^{\prime}} f(x) & =\frac{1}{N} \sum_{b \in B} e^{-2 \pi i l \cdot \tau_{b}(x)} e^{2 \pi i l^{\prime} \tau_{b}(x)} f\left(R \tau_{b}(x)-b\right) \\
& =\left(\frac{1}{N} \sum_{b \in B} e^{2 \pi i\left(l^{\prime}-l\right) \cdot R^{-1} b}\right) e^{2 \pi i\left(l^{\prime}-l\right) \cdot R^{-1} x} f(x) .
\end{aligned}
$$


Therefore $S_{l}^{*} S_{l^{\prime}}=\delta_{l, l^{\prime}} I_{L^{2}\left(\mu_{B}\right)}$ if and only if the matrix in (1.9) is unitary.

Also,

$$
A:=\sum_{l \in L} S_{l} S_{l}^{*} f(x)=\sum_{l \in L} e^{2 \pi i l \cdot x} \frac{1}{N} \sum_{b \in B} e^{-2 \pi i l \cdot \tau_{b}(\mathcal{R} x)} f\left(\tau_{b}(\mathcal{R} x)\right) .
$$

If $x \in \tau_{b_{0}}\left(X_{B}\right)$, then $\tau_{b}(\mathcal{R} x)=x+b-b_{0}$ and further we have

$$
A=\sum_{b \in B} f\left(x+b-b_{0}\right) \frac{1}{N} \sum_{l \in L} e^{2 \pi i l \cdot\left(b-b_{0}\right)} .
$$

If $(B, L)$ is a Hadamard pair, then we get $A=f(x)$, which proves the other Cuntz relation

$$
\sum_{l \in L} S_{l} S_{l}^{*}=I_{L^{2}\left(\mu_{B}\right)}
$$

Definition 1.6. Suppose $(B, L)$ form a Hadamard pair. We denote by $R^{T}$, the transpose of the matrix $R$. We consider the dual IFS

$$
\tau_{l}^{(L)}(x)=\left(R^{T}\right)^{-1}(x+l), \quad\left(x \in \mathbb{R}^{d}, l \in L\right) .
$$

We denote the attractor of this IFS by $X_{L}$ and its invariant measure by $\mu_{L}$.

To simplify the notation, we will use $\tau_{l}$ instead of $\tau_{l}^{(L)}$, and make the convention that when the subscript is an $l$, then we refer to $\tau_{l}^{(L)}$, and when the subscript is a $b$ we refer to $\tau_{b}$.

Definition 1.7. For the set $B$ define the function

$$
\chi_{B}(x)=\frac{1}{N} \sum_{b \in B} e^{2 \pi i b \cdot x}, \quad\left(x \in \mathbb{R}^{d}\right) .
$$

The transfer operator $R_{B, L}$ is defined on functions $f$ on $\mathbb{R}^{d}$ by

$$
\begin{aligned}
\left(R_{B, L} f\right)(x) & =\sum_{l \in L}\left|\chi_{B}\left(\tau_{l}(x)\right)\right|^{2} f\left(\tau_{l}(x)\right) \\
& =\sum_{l \in L}\left|\chi_{B}\left(\left(R^{T}\right)^{-1}(x+l)\right)\right|^{2} f\left(\left(R^{T}\right)^{-1}(x+l)\right) .
\end{aligned}
$$

The operators $S_{l}^{*}$ behave well on exponential functions: the next lemma will be helpful for our computations.

Lemma 1.8. The following assertions hold:

(i) For all $l \in L$ and $t \in \mathbb{R}^{d}$,

$$
S_{l}^{*} e_{t}=e_{-\tau_{l}^{(L)}(-t)} \chi_{B}\left(-\tau_{l}^{(L)}(-t)\right) .
$$

(ii) Let $\mathcal{H}$ be a reducing subspace of $L^{2}\left(\mu_{B}\right)$ for the representation $\left(S_{l}\right)_{l \in L}$. If $e_{t} \in \mathcal{H}$ and $\chi_{B}\left(-\tau_{l}^{(L)}(-t)\right) \neq 0$, then $e_{-\tau_{l}^{(L)}(-t)} \in \mathcal{H}$. 
Proof. We have

$$
\begin{aligned}
\left(S_{l}^{*} e_{t}\right)(x) & =\frac{1}{N} \sum_{b \in B} e^{-2 \pi i l \cdot R^{-1}(x+b)} e^{2 \pi i t \cdot R^{-1}(x+b)}=\frac{1}{N} \sum_{b \in B} e^{-2 \pi i\left(R^{T}\right)^{-1}(-t+l) \cdot(x+b)} \\
& =e^{-2 \pi i\left(R^{T}\right)^{-1}(-t+l) \cdot x} \frac{1}{N} \sum_{b \in B} e^{-2 \pi i\left(R^{T}\right)^{-1}(-t+l) \cdot b} \\
& =e^{-2 \pi i \tau_{l}^{(L)}(-t) \cdot x} \chi_{B}\left(-\tau_{l}^{(L)}(t)\right)=e_{-\tau_{l}^{(L)}(x)} \chi_{B}\left(-\tau_{l}^{(L)}(-t)\right) .
\end{aligned}
$$

(ii) follows from (i).

Definition 1.9. We will use the following notation for a finite word $w=w_{1} \ldots w_{n} \in$ $L^{n}$,

$$
S_{w}=S_{w_{1}} \ldots S_{w_{n}}
$$

Proposition 1.10. For a subspace $H$ of $L^{2}\left(\mu_{B}\right)$ denote by $P_{H}$ the orthogonal projection onto $H$. Let $\mathcal{K}$ be a subspace which is invariant for all the maps $S_{l}^{*}$, $l \in L$, i.e., $S_{l}^{*} \mathcal{K} \subset \mathcal{K}$ for all $l \in L$. Let $\mathcal{K}_{0}:=\mathcal{K}$,

$$
\begin{gathered}
\mathcal{K}_{n}:=\overline{\operatorname{span}}\left\{S_{w} \mathcal{K}: w \in L^{n}\right\}, \\
\mathcal{K}_{\infty}:=\overline{\operatorname{span}}\left\{S_{w} \mathcal{K}: w \in L^{n}, n \in \mathbb{N}\right\} .
\end{gathered}
$$

Then

(i) For all $n \in \mathbb{N}, \mathcal{K}_{n}$ is invariant for $S_{l}^{*}, l \in L$ and $S_{l}^{*} \mathcal{K}_{n+1}=\mathcal{K}_{n}$ for all $l \in L, n \geq 0$.

(ii) $P_{\mathcal{K}_{n}} \leq P_{\mathcal{K}_{n+1}}$ for all $n \in \mathbb{N}$.

(iii) For all $n \in \mathbb{N}$,

$$
P_{\mathcal{K}_{n+1}}=\alpha\left(P_{\mathcal{K}_{n}}\right)=\sum_{l \in L} S_{l} P_{\mathcal{K}_{n}} S_{l}^{*}
$$

(iv) The projections $P_{\mathcal{K}_{n}}$ converge to $P_{\mathcal{K}_{\infty}}$ in the strong operator topology.

Proof. (i) Take $w=w_{1} \ldots w_{n} \in L^{n}$ and $k \in \mathcal{K}$. Then for any $l_{0} \in L$,

$$
S_{l_{0}}^{*} S_{w_{1}} S_{w_{2} \ldots w_{n}} k=\delta_{l_{0} w_{1}} S_{w_{2} \ldots w_{n}} k=\delta_{l_{0} w_{1}} \sum_{l \in L} S_{w_{2} \ldots w_{n}} S_{l} S_{l}^{*} k .
$$

But $S_{l}^{*} k \in \mathcal{K}$ so $S_{l_{0}}^{*} S_{w} k \in \mathcal{K}_{n}$.

This computation implies also that $S_{l_{0}}^{*} \mathcal{K}_{n} \subset \mathcal{K}_{n-1}$. The other inclusion follows from the Cuntz relations, and this implies (i).

(ii) is immediate from (i).

Since $\mathcal{K}_{n}$ is also invariant under the maps $S_{l}^{*}$, it is enough to prove (i) for $n=0$. Take $l_{0} \in L$ and $k \in \mathcal{K}$. Then

$$
\alpha\left(P_{\mathcal{K}}\right) S_{l_{0}} k=\sum_{l \in L} S_{l} P_{\mathcal{K}} S_{l}^{*} S_{l_{0}} k=S_{l_{0}} P_{\mathcal{K}} k=S_{l_{0}} k .
$$

Therefore $\mathcal{K}_{1}$ is contained in the range of the projection $\alpha\left(P_{\mathcal{K}}\right)$.

Also, for any $\alpha\left(P_{\mathcal{K}}\right) v=\sum_{l \in L} S_{l} P_{\mathcal{K}} S_{l}^{*} v \in \mathcal{K}_{1}$ since $P_{\mathcal{K}} S_{l}^{*} v \in \mathcal{K}$.

(iv) is clear since $\cup_{n} \mathcal{K}_{n}$ spans $\mathcal{K}_{\infty}$.

Definition 1.11. For an operator $T$ on $L^{2}\left(\mu_{B}\right)$ define the following function

$$
h_{T}(t)=\left\langle T e_{-t}, e_{-t}\right\rangle, \quad\left(t \in \mathbb{R}^{d}\right) .
$$


Proposition 1.12. Let $T$ be an operator on $L^{2}\left(\mu_{B}\right)$. Then

$$
h_{\alpha(T)}=R_{B, L} h_{T} .
$$

The function $h_{T}$ is entirely analytic.

Proof. We have, with Lemma 1.8

$$
\begin{aligned}
h_{\alpha(T)}(t) & =\left\langle\sum_{l \in L} S_{l} T S_{l}^{*} e_{-t}, e_{-t}\right\rangle=\sum_{l \in L}\left\langle T S_{l}^{*} e_{-t}, S_{l}^{*} e_{-t}\right\rangle \\
& =\sum_{l \in L}\left|\chi_{B}\left(\tau_{l}(t)\right)\right|^{2}\left\langle T e_{-\tau_{l}(t)}, e_{-\tau_{l}(t)}\right\rangle \\
& =\sum_{l \in L}\left|\chi_{B}\left(\tau_{l}(t)\right)\right|^{2} h_{T}\left(\tau_{l}(t)\right)=R_{B, L} h_{T}(t) .
\end{aligned}
$$

Since the operator $T$ is bounded, it is easy to check that the function $h_{T}$ is entirely analytic.

Corollary 1.13. Using the notations above:

(i) If $A$ is a bounded operator in the commutant of the representation $\left(S_{l}\right)_{l \in L}$, then the function $h_{A}$ is an entire analytic harmonic function for the transfer operator $R_{B, L}$, i.e.,

$$
R_{B, L} h_{A}=h_{A} .
$$

(ii) Suppose $\mathcal{K}$ is a subspace which is invariant under all the maps $S_{l}^{*}, l \in L$. Then the function $h_{P_{\mathcal{K}}}$ is an entire analytic subharmonic function for the transfer operator $R_{B, L}$ i.e.,

$$
R_{B, L} h_{P_{\mathcal{K}}} \geq h_{P_{\mathcal{K}}} .
$$

Proof. If $A$ commutes with the representation, then $\alpha(A)=\sum_{l} S_{l} A S_{l}^{*}=A \sum_{l} S_{l} S_{l}^{*}$ $=A$. If $\mathcal{K}$ is invariant under the maps $S_{l}^{*}$, then $\alpha\left(P_{\mathcal{K}}\right) \geq P_{\mathcal{K}}$ by Proposition 1.10 . Then the corollary follows directly from Proposition 1.12

Corollary 1.14. Let $\mathcal{K}$ be a subspace of $L^{2}\left(\mu_{B}\right)$ which is invariant under all the maps $S_{l}^{*}, l \in L$. With the notation in Proposition 1.10, the following limit exists uniformly on compact sets:

$$
\lim _{n \rightarrow \infty} R_{B, L}^{n} h_{P_{\mathcal{K}}}=h_{P_{\mathcal{K}}} .
$$

Proof. With Propositions 1.12 and 1.10 we have

$$
R_{B, L}^{n} h_{P_{\mathcal{K}}}=h_{\alpha^{n}\left(P_{\mathcal{K}}\right)}=h_{P_{\mathcal{K}_{n}}} .
$$

Since $P_{\mathcal{K}_{n}}$ converges in the strong operator topology to $P_{\mathcal{K}_{\infty}}$, we obtain that $h_{P_{\mathcal{K}_{n}}}$ converges to $h_{P_{\mathcal{K}_{\infty}}}$ pointwise. Using Corollary 1.13, we see that this is an increasing sequence of functions; then, with Dini's theorem we obtain the conclusion.

\section{REPRESENTATIONS ASSOCiATED TO MINIMAL WORDS}

We now turn to a dynamical systems feature which determines both the algorithmic and the analytic part of the problem of $L^{2}(\mu)$. This feature in turns divides up into two parts, periodic and non-periodic. The precise meaning of these terms is fleshed out in Definitions 2.1 and 2.2 (in the present section), and in Definition 4.2 inside the paper. In both cases, we deal with a random walk-dynamical system. The first case is especially easy to understand in terms of a natural encoding with 
finite and infinite code-words. By contrast, the second case involves invariant sets (for the walk, Definition 4.2) which can have quite subtle fractal properties. Some of the possibilities (case 2) are illustrated by examples in section 4 .

We begin with a discussion of the minimal words in the encoding for case 1 .

Definition 2.1. Consider a finite word over a finite alphabet $L$ with $\# L=N$. We say that $w$ is minimal if there is no word $u$ such that $w=\underbrace{u u \ldots u}_{p \text { times }}$ for some $p \geq 2$.

We denote by $\underline{w}=w w \ldots$, the infinite word obtained by the repetition of $w$ infinitely many times. For two words $u, w$ with $u$ finite, we denote by $u w$ the concatenation of the two words.

We define the shift $\sigma$ on infinite words $\sigma\left(\omega_{1} \omega_{2} \ldots\right)=\omega_{2} \omega_{3} \ldots$

Definition 2.2. Let $w$ be a minimal word. Let $\Gamma(w)$ be the set of infinite words over the alphabet $L$ that end in an infinite repetition of the word $w$, i.e.,

$$
\Gamma(w):=\{u \underline{w}: u \text { is a finite word over } L\} .
$$

Consider the Hilbert space

$$
l^{2}(\Gamma(w)):=\left\{x: \Gamma(w) \rightarrow \mathbb{C}: \sum_{\alpha \in \Gamma(w)}|x(\alpha)|^{2}<\infty\right\} .
$$

The inner product is defined by

$$
\langle x, y\rangle=\sum_{\alpha \in \Gamma(w)} x(\alpha) \overline{y(\alpha)} .
$$

We will use the Dirac notation for vectors in $l^{2}(\Gamma(w))$ : for $\alpha \in \Gamma(w)$

$$
|\alpha\rangle=\delta_{\alpha}, \quad \delta_{\alpha}(\xi)= \begin{cases}1, & \xi=\alpha \\ 0, & \xi \neq \alpha .\end{cases}
$$

We define the represetation $\rho_{w}$ of the Cuntz algebra $\mathcal{O}_{N}$ by

$$
\rho_{w}\left(S_{l}\right)|\alpha\rangle=|l \alpha\rangle, \quad\left(\alpha \in l^{2}(\Gamma(w))\right)
$$

Moreover, if $u, v$ are vectors in a Hilbert space, we will use the notation $|u\rangle\langle v|$ for the corresponding rank-one operator.

Theorem 2.3. The following assertions hold:

(i) The operators in (2.2) defines an irreducible representation of the Cuntz algebra $\mathcal{O}_{N}$.

(ii) Define the subspace $\mathcal{K}_{w}$ spanned by the vectors $\left\{|\underline{w}\rangle,|\sigma(\underline{w})\rangle, \ldots,\left|\sigma^{p-1}(\underline{w})\right\rangle\right\}$, where $p$ is the length of $w$. Then $\mathcal{K}_{w}$ is invariant for the operators $\rho_{w}\left(S_{l}\right)^{*}$, $l \in L$ and it is cyclic for the representation $\rho_{w}$.

(iii) If $w$ and $w^{\prime}$ are two minimal words that are not a cyclic permutation of each other, then the representations $\rho_{w}$ and $\rho_{w^{\prime}}$ are disjoint.

Proof. It is easy to check that

$$
\rho_{w}\left(S_{l}\right)^{*}|\alpha\rangle=\left\{\begin{array}{cl}
0, & \text { if } \alpha_{1} \neq l, \\
|\sigma(\alpha)\rangle, & \text { if } \alpha_{1}=l,
\end{array}\right.
$$

for $\alpha=\alpha_{1} \alpha_{2} \ldots$. From this it follows after a simple computation that $\rho_{w}$ is a representation of the Cuntz algebra. 
Since $\sigma^{p}(\underline{w})=\underline{w}$, it follows that $\rho_{w}\left(S_{l}\right)^{*} \mathcal{K}_{w} \subset \mathcal{K}_{w}$. Since every word in $\Gamma(w)$ ends in $\underline{w}$, it can be easily seen that $\mathcal{K}_{w}$ is cyclic for the representation.

It remains to check that the representation is irreducible and assertion (iii). For this we will use Theorem from [BJKW00, applied to our situation (see also BJ97b, BJ97a]):

Theorem 2.4. There is a bijective correspondence between:

(i) operators $A$ that intertwine the representations $\rho_{w}$ and $\rho_{w^{\prime}}$, i.e.,

$$
\rho_{w^{\prime}}\left(S_{l}\right) A=A \rho_{w}\left(S_{l}\right), \quad(l \in L) ;
$$

(ii) fixed points of the map

$$
\Phi(C)=\sum_{l \in L} V_{l}^{\prime} C V_{l}^{*}, \quad\left(C \in \mathcal{B}\left(\mathcal{K}_{w}, \mathcal{K}_{w^{\prime}}\right)\right),
$$

where $V_{l}=P \rho_{w}\left(S_{l}\right) P, V_{l}^{\prime}=P^{\prime} \rho_{w^{\prime}}\left(S_{l}\right) P^{\prime}, l \in L$, with $P=$ projection onto $\mathcal{K}_{w}, P^{\prime}=$ projection onto $\mathcal{K}_{w^{\prime}}$. The correspondence from (i) to (ii) is given by

$$
C=P^{\prime} A P \text {. }
$$

Returning to the proof of Theorem 2.3, we will compute the fixed points of the $\operatorname{map} \Phi$.

Let $u_{j}=\left|\sigma^{j}(\underline{w})\right\rangle, j=0, \ldots, p-1$, where $p=$ length of $w$, and let $u_{i}^{\prime}=\left|\sigma^{i}\left(\underline{w}^{\prime}\right)\right\rangle$, $i=0, \ldots, p^{\prime}-1$, where $p^{\prime}=$ length of $w^{\prime}$. The space $\mathcal{B}\left(\mathcal{K}_{w}, \mathcal{K}_{w^{\prime}}\right)$ is spanned by the rank one operators $\left|u_{i}^{\prime}\right\rangle\left\langle u_{j}\right|$. We have

$$
\Phi\left(\left|u_{i}^{\prime}\right\rangle\left\langle u_{j}\right|\right)=\sum_{l \in L}\left|V_{l}^{\prime} u_{i}^{\prime}\right\rangle\left\langle V_{l} u_{j}\right| .
$$

But

$$
V_{l} u_{j}=P \rho_{w}\left(S_{l}\right) u_{j}=P\left|l \sigma^{j}(\underline{w})\right\rangle=P\left|l w_{j+1} w_{j+2} \ldots w_{p} \underline{w}\right\rangle=\delta_{l, w_{j}}\left|\sigma^{j-1}(\underline{w})\right\rangle
$$

(we use here a notation $\bmod p$, or $\bmod p^{\prime}$ when required, so $\sigma^{-1}(\underline{w})$ will mean $\left.\sigma^{p-1}(\underline{w})\right)$.

Then

$$
\Phi\left(\left|u_{i}^{\prime}\right\rangle\left\langle u_{j}\right|\right)=\sum_{l \in L} \delta_{l, w_{i}^{\prime}} \delta_{l, w_{j}}\left|\sigma^{i-1}\left(\underline{w}^{\prime}\right)\right\rangle\left\langle\sigma^{j-1}(\underline{w})\left|=\delta_{w_{i}^{\prime}, w_{j}}\right| \sigma^{i-1}\left(\underline{w}^{\prime}\right)\right\rangle\left\langle\sigma^{j-1}(\underline{w})\right| .
$$

Now suppose $C=\sum_{i=0}^{p^{\prime}-1} \sum_{j=0}^{p-1} c_{i, j}\left|u_{i}^{\prime}\right\rangle\left\langle u_{j}\right|$ is a fixed point for $\Phi$. Then

$$
\sum_{i=0}^{p^{\prime}-1} \sum_{j=0}^{p-1} c_{i, j}\left|u_{i}^{\prime}\right\rangle\left\langle u_{j}\left|=\sum_{i, j} c_{i, j} \delta_{w_{i}^{\prime}, w_{j}}\right| u_{i-1}^{\prime}\right\rangle\left\langle u_{j-1}\right| .
$$

Then

$$
c_{i, j}=c_{i+1, j+1} \delta_{w_{i+1}^{\prime}, w_{j+1}} \text {, for all } i, j .
$$

Thus, $c_{i, j} \neq 0$ only if $w_{i+k}^{\prime}=w_{j+k}$ for all $k$, which means that $\sigma^{i-j}\left(\underline{w}^{\prime}\right)=\underline{w}$. But this implies, since $w, w^{\prime}$ are minimal, that $w$ is a cyclic permutation of $w^{\prime}$. Thus, if $w, w^{\prime}$ are not cyclic permutations of each other, then the only fixed point of $\Phi$ is $C$, and with Theorem 2.4 this implies that there are no nonzero intertwining operators.

To check that the representation $\rho_{w}$ is irreducible, we use the same computation, now with $w=w^{\prime}$. We saw that $c_{i, j} \neq 0$ only if $\sigma^{i-j}\left(\underline{w}^{\prime}\right)=\underline{w}$. But since $|i-j|<p$ and $w$ is minimal this implies that $i=j$. In the case $i=j$, the same computation 
implies that $c_{i, i}=c_{i+k, i+k}$, therefore $C$ is a scalar multiple of the identity. Using again Theorem 2.4. we obtain that the only operators in the commutant of $\rho_{w}$ are scalar multiples of the identity.

\section{Representations Associated to EXtREME CYCLES}

We mentioned in section 2 , that the algorithmic and the analytic part of the problem of $L^{2}(\mu)$ involves a dynamical system. Its nature is divided into two parts, periodic and nonperiodic. Case 1 has an algorithmic part (code-words), and an analytic part taking the form of extreme cycles, and their associated representations. This is worked out below.

Definition 3.1. A finite set of distinct points $C=\left\{x_{0}, x_{1}, \ldots, x_{p-1}\right\}$ is called an $L$-cycle if there exist $l_{0}, \ldots, l_{p-1} \in L$ such that

$$
\tau_{l_{0}}\left(x_{0}\right)=x_{1}, \tau_{l_{1}}\left(x_{1}\right)=x_{2}, \ldots, \tau_{l_{p-2}}\left(x_{p-2}\right)=x_{p-1}, \tau_{l_{p-1}}\left(x_{p-1}\right)=x_{0} .
$$

We call $w(C):=l_{0} l_{1} \ldots l_{p-1}$ the word of the cycle $C$. The points in $C$ are called $L$-cycle points.

An $L$-cycle is called $B$-extreme if

$$
\left|\chi_{B}(x)\right|=1 \text {, for all } x \in C .
$$

Theorem 3.2 ([DJ07, Theorem 4.1]). Under the conditions above, assume $(B, L)$ is a Hadamard pair. Suppose there exist d linearly independent vectors in the set

$$
\Gamma(B):=\left\{\sum_{k=0}^{n} R^{k} b_{k}: b_{k} \in B, n \in \mathbb{N}\right\} .
$$

Define

$$
\Gamma(B)^{\circ}:=\left\{x \in \mathbb{R}^{d}: \beta \cdot x \in \mathbb{Z} \text { for all } \beta \in \Gamma(B)\right\} .
$$

Then $\Gamma(B)^{\circ}$ is a lattice that contains $\mathbb{Z}^{d}$, is invariant under $R^{T}$, and if $l, l^{\prime} \in L$ with $l-l^{\prime} \in R^{T} \Gamma(B)^{\circ}$, then $l=l^{\prime}$. Moreover,

$$
\Gamma(B)^{\circ} \cap X_{L}=\bigcup\{C: C \text { is a B-extreme L-cycle }\} .
$$

Definition 3.3. Let $(B, L)$ be a Hadamard pair. We say that the Hadamard pair is regular if the IFS $\left(\tau_{b}\right)_{b \in B}$ has no overlap and there exist $d$ linearly independent vectors in the set $\Gamma(B)$ defined by (3.2).

Define the maps $\sigma_{l}$ on $\mathbb{R}^{d}$ by

$$
\sigma_{l}(x)=R^{T} x+l, \quad\left(x \in \mathbb{R}^{d}, l \in L\right) .
$$

A subset $\Lambda$ of $\mathbb{R}^{d}$ is called invariant if $\sigma_{l}(\Lambda) \subset \Lambda$ for all $l \in L$.

Theorem 3.4. Let $(B, L)$ be a regular Hadamard pair and let $C$ be a $B$-extreme $L$ cycle. Let $\Lambda(C)$ be the smallest invariant set that contains $-C$. Define the subspace of $L^{2}\left(\mu_{B}\right)$ :

$$
\mathcal{H}(C):=\overline{\operatorname{span}}\left\{e_{\lambda}: \lambda \in \Lambda(C)\right\}
$$

Then:

(i) The space $\mathcal{H}(C)$ is a reducing subspace for the representation $\left(S_{l}\right)_{l \in L}$ of the Cuntz algebra $\mathcal{O}_{N}$. 
(ii) The exponential function $\left\{e_{\lambda}: \lambda \in \Lambda(C)\right\}$ form an orthonormal basis for $\mathcal{H}(C)$ and

$$
S_{l}\left(e_{\lambda}\right)=e_{\sigma_{l}(\lambda)}, \quad(\lambda \in \Lambda(C), l \in L) .
$$

(iii) If $C_{1}$ and $C_{2}$ are two distinct $B$-extreme $L$-cycles, then the subspaces $\mathcal{H}\left(C_{1}\right)$ and $\mathcal{H}\left(C_{2}\right)$ are orthogonal.

(iv) Let $w(C)$ be the word of the cycle $C$. The restriction of the representation $\left(S_{l}\right)_{l \in L}$ to $\mathcal{H}(C)$ is equivalent to the representation $\rho_{w(C)}$ on $l^{2}(\Gamma(w(C))$ from Theorem 2.3. The isomorphism can be defined as follows: define the encoding map $\epsilon_{C}: \Lambda(C) \rightarrow \Gamma(w(C)), \epsilon_{C}(\lambda)=\alpha_{1} \alpha_{2} \ldots$, where $\alpha_{1}, \alpha_{2}, \ldots$ are uniquely defined by the condition

$$
S_{\alpha_{n}}^{*} \ldots S_{\alpha_{1}}^{*} e_{\lambda} \neq 0, \quad(n \in \mathbb{N}) .
$$

Then the map $W_{C}: \mathcal{H}(C) \rightarrow l^{2}(\Gamma(w(C))$

$$
W_{C}\left(e_{\lambda}\right)=\left|\epsilon_{C}(\lambda)\right\rangle
$$

defines an isometric isomorphism that intertwines the two representations of $\mathcal{O}_{N}$.

(v) The restrictions of the representation $\left(S_{l}\right)_{l \in L}$ to the subspaces $\mathcal{H}(C)$, where $C$ is any $B$-extreme $L$-cycle, are mutually disjoint irreducible representations of the Cuntz algebra $\mathcal{O}_{N}$.

We begin with some lemmas:

Lemma 3.5. In the hypotheses of Theorem 3.4 we have:

(i) $\Lambda(C) \subset \Gamma(B)^{\circ}$.

(ii) For every $t \in \Gamma(B)^{\circ}$ and $l \in L, S_{l} e_{t}=e_{\sigma_{l}(t)}$.

(iii) If $t=\sigma_{l}\left(t^{\prime}\right)$ for some $l \in L$ and $t^{\prime} \in \Gamma(B)^{\circ}$, then $S_{l^{\prime}}^{*} e_{t}=\delta_{l l^{\prime}} e_{t^{\prime}}$.

(iv) For all $t \in \Lambda(C)$, there exist a unique $t^{\prime} \in \Lambda(C)$ and $l \in L$ such that $t=\sigma_{l}\left(t^{\prime}\right)$. Moreover, if $t \in-C$, then $t^{\prime} \in-C$.

Proof. (i) follows from Theorem 3.2. For (ii), let $t \in \Gamma(B)^{\circ}$ and $x \in \tau_{b}\left(X_{B}\right)$ for some $b \in B$. Then

$$
\left(S_{l} e_{t}\right)(x)=e^{2 \pi i l \cdot x} e^{2 \pi i t \cdot \mathcal{R} x}=e^{2 \pi i l \cdot x} e^{2 \pi i t \cdot\left(R^{T} x-b\right)} .
$$

But $t \in \Gamma(B)^{0}$ implies that $t \cdot b \in \mathbb{Z}$, so

$$
\left(S_{l} e_{t}\right)(x)=e_{\sigma_{l}(t)}(x) .
$$

(iii) If $t=\sigma_{l}\left(t^{\prime}\right)$ with $t^{\prime} \in \Gamma(B)^{\circ}$, then, using (ii),

$$
S_{l^{\prime}}^{*} e_{t}=S_{l^{\prime}}^{*} S_{l} e_{t^{\prime}}=\delta_{l l^{\prime}} e_{t^{\prime}} .
$$

(iv) It is clear that

$$
\Lambda(C)=\left\{\sigma_{l_{n}} \ldots \sigma_{l_{1}} x: x \in-C, l_{1}, \ldots, l_{n} \in L, n \in \mathbb{N}\right\} .
$$

Therefore, for existence, we have to check only that if $x \in-C$, then $x=\sigma_{l}\left(t^{\prime}\right)$ for some $l \in L$ and $t^{\prime} \in-C$. Since $-x \in C$ and $C$ is an $L$-cycle, there exist $l \in L$ and $y \in C$ such that $\left(R^{T}\right)^{-1}(x+l)=y$. This implies that $\sigma_{l}(-y)=-x$, and this proves the assertion.

For uniqueness, suppose $t=\sigma_{l_{1}}\left(t_{1}^{\prime}\right)=\sigma_{l_{2}}\left(t_{2}^{\prime}\right)$ with $l_{1}, l_{2} \in L, t_{1}, t_{2} \in \Lambda(C)$. Then $l_{1}-l_{2}=R^{T}\left(t_{2}^{\prime}-t_{1}^{\prime}\right)$. But since $t_{2}^{\prime}-t_{1}^{\prime} \in \Gamma(B)^{\circ}$, Theorem 3.2 implies that $l_{1}=l_{2}$ and so $t_{1}^{\prime}=t_{2}^{\prime}$. 
Lemma 3.6. Let $(B, L)$ be a regular Hadamard pair. Then:

(i) The Fourier transform of the measure $\mu_{B}$, defined by

$$
\widehat{\mu}_{B}(x)=\int e^{2 \pi i t \cdot x} d \mu_{B}(t), \quad\left(t \in \mathbb{R}^{d}\right)
$$

satisfies the following equality

$$
\widehat{\mu}_{B}(x)=\prod_{n=1}^{\infty} \chi_{B}\left(\left(R^{T}\right)^{-n} x\right), \quad\left(x \in \mathbb{R}^{d}\right) .
$$

(ii) Two exponential functions $e_{t}$ and $e_{t^{\prime}}$ are orthogonal in $L^{2}\left(\mu_{B}\right)$ iff $\widehat{\mu}_{B}(t-$ $\left.t^{\prime}\right)=0$.

(iii) The function $\chi_{B}$ satisfies the following $Q M F$ condition

$$
\sum_{l \in L}\left|\chi_{B}\left(\left(R^{T}\right)^{-1}(x+l)\right)\right|^{2}=1, \quad\left(x \in \mathbb{R}^{d}\right) .
$$

(iv) For all $t \in \Gamma(B)^{\circ}$,

$$
\chi_{B}(x+t)=\chi_{B}(x), \quad\left(x \in \mathbb{R}^{d}\right) .
$$

(v) If $C$ is a B-extreme $L$-cycle, and $x \in C$ with $-x=\sigma_{l_{0}}(-y)$ for some $l_{0} \in L$ and $y \in C$, then

$$
\chi_{B}\left(\left(R^{T}\right)^{-1}(x+l)\right)=0 \text { for all } l \in L, l \neq l_{0} .
$$

Proof. For the proof of (i)-(iii) we refer to DJ06b. For (iv), if $t \in \Gamma(B)^{\circ}$, then $b \cdot t \in \mathbb{Z}$ so $e^{2 \pi i b \cdot t}=1$ for all $b \in B$ and this implies (iv).

For $(\mathrm{v})$, the equality $-x=\sigma_{l_{0}}(-y)$ implies that $\left(R^{T}\right)^{-1}\left(x+l_{0}\right)=y$. Since the cycle is $B$-extreme $\left|\chi_{B}(y)\right|=1$. Then, using (iii), one obtains that $\mid \chi_{B}\left(\left(R^{T}\right)^{-1}(x+\right.$ $l) \mid=0$ for all $l \neq l_{0}$.

Proof of Theorem 3.4. The fact that $\mathcal{H}(C)$ is invariant for all $S_{l}$ and $S_{l}^{*}$ follows from Lemma 3.5 (ii) and (iii).

Next let $C^{\prime}$ be a $B$-extreme $L$-cycle, and let $t \in \Lambda(C), t^{\prime} \in \Lambda\left(C^{\prime}\right)$. Then there exist $x \in C, x^{\prime} \in C^{\prime}, l_{1}, \ldots, l_{n} \in L, l_{1}^{\prime}, \ldots, l_{m}^{\prime} \in L$ such that $t=\sigma_{l_{n}} \ldots \sigma_{l_{1}}(-x)$ and $t^{\prime}=\sigma_{l_{n}^{\prime}} \ldots \sigma_{l_{1}^{\prime}}\left(-x^{\prime}\right)$. Using Lemma 3.5(iv), and composing with a few more $\sigma_{l}$ 's if necessary, we can assume $n=m$. We then have with Lemma 3.5(ii), and then using the Cuntz relations,

$$
\begin{aligned}
\left\langle e_{t}, e_{t^{\prime}}\right\rangle=\left\langle S_{l_{n}} \ldots S_{l_{1}} e_{-x}, S_{l_{n}^{\prime}} \ldots S_{l_{1}^{\prime}} e_{-x^{\prime}}\right\rangle & =\delta_{l_{n} l_{n}^{\prime}} \ldots \delta_{l_{1} l_{1}^{\prime}}\left\langle e_{-x}, e_{-x^{\prime}}\right\rangle \\
& =\delta_{l_{n} l_{n}^{\prime}} \ldots \delta_{l_{1} l_{1}^{\prime}} \widehat{\mu}_{B}\left(x^{\prime}-x\right) .
\end{aligned}
$$

Thus, to prove (ii) and (iii), it is enough to show that $\widehat{\mu}_{B}\left(x^{\prime}-x\right)=0$ for $x \neq x^{\prime}$.

By Lemma 3.5 (iv), $-x=\sigma_{l_{0}}(-y)$ for some $l_{0} \in L$ and $y \in C$, and $-x^{\prime}=\sigma_{l_{0}^{\prime}}\left(-y^{\prime}\right)$ for some $l_{0}^{\prime} \in L$ and $y^{\prime} \in C^{\prime}$. We can assume $l_{0} \neq l_{0}^{\prime}$. If not, then one can compose with a few more $\sigma_{l}$ 's in the argument above until the cycle $C$ uses another digit $l_{0}$ than the cycle $C^{\prime}$.

We have

$$
\begin{aligned}
\chi_{B}\left(\left(R^{T}\right)^{-1}\left(x^{\prime}-x\right)\right)=\chi_{B}\left(\left(R^{T}\right)^{-1}\left(x^{\prime}+l_{0}\right)-\left(R^{T}\right)^{-1}\left(x+l_{0}\right)\right) \\
=\chi_{B}\left(\left(R^{T}\right)^{-1}\left(x^{\prime}+l_{0}\right)-y\right)=\chi_{B}\left(\left(R^{T}\right)^{-1}\left(x^{\prime}+l_{0}\right)\right) \\
\quad \quad \quad\left(\text { because } y \in \Gamma(B)^{\circ} \text { and due to Lemma 3.6(iv) }\right) \\
=0, \quad \quad \quad\left(\text { from Lemma 3.6(v), because } l_{0} \neq l_{0}^{\prime}\right) .
\end{aligned}
$$


The fact that $\epsilon_{C}$ is well defined follows from Lemma 3.5 (iii) and (iv).

To check (iv), we note that

$$
W_{C} S_{l} e_{\lambda}=W_{C} e_{\sigma_{l}(\lambda)}=\left|\epsilon_{C}\left(\sigma_{l}(\lambda)\right)\right\rangle=\left|l \epsilon_{C}(\lambda)\right\rangle=\rho_{w(C)}\left(S_{l}\right)\left|\epsilon_{C}(\lambda)\right\rangle=\rho_{w(C)}\left(S_{l}\right) W_{C} e_{\lambda} .
$$

Thus $W_{C}$ is intertwining.

Also, we have $\tau_{w(C)_{1}} x_{0}=x_{1}$ so $-x_{0}=R^{T}\left(-x_{1}\right)+w(C)_{1}$, and therefore $S_{w(C)_{1}} e_{-x_{1}}$ $=e_{-x_{0}}$ so $S_{w(C)_{1}}^{*} e_{-x_{0}}=e_{-x_{1}}$. By induction, we get that $\epsilon_{C}\left(-x_{0}\right)=w(C)$.

From the definition of $\Lambda(C)$ we see that for each $\lambda \in \Lambda(C)$, there exists a point $x_{k}$ in $-C$ and some digits $l_{1}, \ldots, l_{n}$ such that $\lambda=\sigma_{l_{1}} \ldots \sigma_{l_{n}}\left(-x_{k}\right)$. Composing with a few more $\sigma_{l}$ 's we can assume $x_{k}=x_{0}$, so $\lambda=\sigma_{l_{1}} \ldots \sigma_{l_{n}}\left(-x_{0}\right)$.

Then

$$
S_{l_{1}} e_{\sigma_{l_{2}} \ldots \sigma_{l_{n}}\left(-x_{0}\right)}=e_{\lambda} \text {, so } S_{l_{1}}^{*} e_{\lambda}=e_{\sigma_{l_{2}} \ldots \sigma_{l_{n}}\left(-x_{0}\right)} .
$$

By induction, we get that $\epsilon_{C}(\lambda)=l_{1} \ldots l_{n} w(C)$.

This proves, in particular, that $\epsilon_{C}$ is surjective, so $W_{C}$ is onto.

Note that we cannot have $\lambda=\sigma_{l_{1}} \lambda_{1}=\sigma_{l_{1}^{\prime}} \lambda_{1}^{\prime}$ for $l_{1} \neq l_{1}^{\prime}$ and $\lambda_{1}, \lambda_{1}^{\prime} \in \Lambda(C)$, because in this case $l_{1}+R^{T} \lambda_{1}=l_{1}^{\prime} R^{T} \lambda_{1}^{\prime}$ so $l_{1}-l_{1}^{\prime} \in R^{T} \Gamma(B)^{\circ}$, and with Theorem 3.2. it follows that $l_{1}=l_{1}^{\prime}$. Therefore, the encoding $\epsilon_{C}$ is well defined.

To see that $\epsilon_{C}$ is injective, suppose $\epsilon_{C}(\lambda)=\epsilon_{C}\left(\lambda^{\prime}\right)=l_{1} \ldots l_{n} w(C)$ then $\lambda=$ $\sigma_{l_{1}} \ldots \sigma_{l_{n}}\left(-x_{0}\right)=\lambda^{\prime}$. Hence $W_{C}$ maps an orthonormal basis to an orthonormal basis and therefore it is an isometric isomorphism.

The last statement in the theorem follows now from Theorem 2.3 .

Corollary 3.7. If the dimension $d=1$, then the decomposition of the representation $\left(S_{l}\right)_{l \in L}$ into irreducible subrepresentation is given by

$$
L^{2}\left(\mu_{B}\right)=\bigoplus\{\mathcal{H}(C): C \text { is a B-extreme L-cycle }\} .
$$

The commutant is finite-dimensional and abelian.

Proof. From DJ06b we know that, when the dimension $d=1$, then the union of the sets $\Lambda(C)$ is a spectrum for $\mu_{B}$, therefore the corresponding exponentials form a complete orthonormal set in $L^{2}\left(\mu_{B}\right)$. Everything then follows from Theorem 3.4 .

\section{BEYOND CYCLES}

While in simple cases, the harmonic analysis of $L^{2}(\mu)$ may be accounted for by cycles (sections 2 and 3 ), there is a wider class involving more noncyclic invariant sets (Definition 4.2). These are studied below. We show (Theorem 4.6) that even in the noncyclic case we still get an associated splitting of the Hilbert space $L^{2}(\mu)$ into orthogonal closed subspaces. We further show by examples that the invariant sets can have quite subtle fractal properties. Some of the possibilities (case 2) are illustrated in Example 4.7 and the results following it. This approach to invariant sets was first introduced in CR90, CCR96, CHR97.

Definition 4.1. For a finite word $w=l_{1} \ldots l_{n}$ we use the notation

$$
\begin{gathered}
\tau_{w}=\tau_{l_{n}} \ldots \tau_{l_{1}} \\
\chi_{B}^{(n)}(x)=\chi_{B}(x) \chi_{B}\left(R^{T} x\right) \ldots \chi_{B}\left(\left(R^{T}\right)^{n-1} x\right), \quad\left(x \in \mathbb{R}^{d}\right) .
\end{gathered}
$$


For the operators $\left(S_{l}\right)_{l \in L}$ defined in (1.10) and any word $w=l_{1} \ldots l_{n}$ we use the notation

$$
S_{w}=S_{l_{1}} \ldots S_{l_{n}} .
$$

Definition 4.2. A subset $M$ of $\mathbb{R}^{d}$ is called $L$-invariant if for all $x \in M$ and all $l \in L$, if $\chi_{B}\left(\tau_{l}(x)\right) \neq 0$, then $\tau_{l} x \in M$. We say that the transition from $x$ to $\tau_{l} x$ is possible if $\chi_{B}\left(\tau_{l} x\right) \neq 0$. For $x \in \mathbb{R}^{d}$ we denote by $O(x)$, the orbit of $x$, that is, the set of all points $y \in \mathbb{R}^{d}$ such that there exist $y_{1}=x_{0}, \ldots, y_{n}=y$ such that the transition from $y_{i}$ to $y_{i+1}$ is possible. Two $L$-invariant sets $M_{1}, M_{2}$ are called separated if $\operatorname{dist}\left(M_{1}, M_{2}\right)>0$. (Here dist denotes a distance under which all maps $\tau_{l}$ are strict contractions, and which generates the Euclidian topology on $\mathbb{R}^{d}$.) A family $\left\{M_{i}: i=1, \ldots, n\right\}$ of closed $L$-invariant sets is called complete if for any closed $L$-invariant set $M$,

$$
M \cap \bigcup_{i=1}^{n} M_{i} \neq \emptyset .
$$

A closed $L$-invariant set $M$ is called minimal if for any closed $L$-invariant set $M^{\prime} \subset M, M^{\prime}=M$.

Here are a few simple properties of $L$-invariant sets.

Proposition 4.3. The following assertions hold:

(i) If $M$ is an L-invariant set, then the closure $\bar{M}$ is also L-invariant.

(ii) Any B-extreme L-cycle is an L-invariant set.

(iii) For any closed minimal $L$-invariant set $M$ and any $x \in M, M=\overline{O(x)}$. Also, $M$ is contained in the attractor $X_{L}$ of the IFS $\left(\tau_{l}\right)_{l \in L}$.

(iv) The family of minimal closed L-invariant sets is a finite, complete family of separated L-invariant sets.

Proof. (i) Take $x \in \bar{M}$ and $l \in L$ such that $\chi_{B}\left(\tau_{l} x\right) \neq 0$. There exists a sequence in $x_{n} \in M$ that converges to $x$. For $n$ large enough $\chi_{B}\left(\tau_{l} x_{n}\right) \neq 0$. Since $M$ is invariant, it follows that $\tau_{l} x_{n} \in M$ so $\tau_{l} x \in \bar{M}$. Therefore $\bar{M}$ is invariant.

(ii) Since $(B, L)$ form a Hadamard pair, it follows (see [DJ06b]) that we have the equality

$$
\sum_{l \in L}\left|\chi_{B}\left(\tau_{l} x\right)\right|^{2}=1, \quad\left(x \in \mathbb{R}^{d}\right) .
$$

Let $C=\left\{x_{0}, x_{1}, \ldots, x_{p-1}\right\}$ be a $B$-extreme $L$-cycle, with $\tau_{l_{0}} x_{0}=x_{1}, \ldots, \tau_{l_{p-1}} x_{p-1}$ $=x_{0}$. Since $\left|\chi_{B}\left(x_{1}\right)\right|=1$, it follows that $\chi_{B}\left(\tau_{l} x_{0}\right) \neq 0$ for $l \neq 0$. So the only possible transition is from $x_{0}$ to $x_{1}$. Similarly for the other points of the cycle. Therefore $C$ is $L$-invariant.

(iii) Clearly,m $O(x)$ is an $L$-invariant set. Since $M$ is minimal and $M$ contains $O(x)$ it follows that $M=\overline{O(x)}$. Also, $X_{L}$ is clearly $L$-invariant. Therefore $M \cap X_{L}$ is $L$-invariant. So $M \cap X_{L}$ is either $M$ or $\emptyset$. We will prove that it cannot be $\emptyset$.

Take $x \in M$. Using (4.1), we see that a transition is always possible, so we can construct $l_{1}, l_{2}, \ldots$ such that $y_{n}:=\tau_{l_{n}} \ldots \tau_{l_{1}} x \in O(x)$ for all $n$. But since $X_{L}$ is the attractor of the IFS $\left(\tau_{l}\right)_{l \in L}$ it follows that $\operatorname{dist}\left(y_{n}, X_{L}\right) \rightarrow 0$. So $\bar{O}(x) \cap X_{L} \neq$ and therefore $M \cap X_{L} \neq \emptyset$.

(iv) First we prove that any two distinct minimal closed $L$-invariant sets $M_{1}, M_{2}$ are separated. Since $M_{1}, M_{2}$ are contained in $X_{L}$, they are compact, so it is enough 
to show that they are disjoint. But $M_{1} \cap M_{2}$ is also $L$-invariant and closed so $M_{1} \cap M_{2}=\emptyset$ due to minimality.

Next we prove that actually there exists a $\delta>0$ that does not depend on $M_{1}, M_{2}$ such that $\operatorname{dist}\left(M_{1}, M_{2}\right) \geq \delta$.

Since $\chi_{B}$ is uniformly continuous on $X_{L}$, there exists a $\delta>0$ such that if $\operatorname{dist}(x, y)<\delta$, then $\left|\chi_{B}(x)-\chi_{B}(y)\right|<1 / 2 N$.

Suppose there exist two points $x_{0} \in M_{1}, y_{0} \in M_{2}$ such that $\operatorname{dist}(x, y)<$ $\delta$. From (4.1), there exists $l_{0} \in L$ such that $\left|\chi_{B}\left(\tau_{l_{0}} x_{0}\right)\right| \geq 1 / N$. Then, since $\operatorname{dist}\left(\tau_{l_{0}} x_{0}, \tau_{l_{0}} y_{0}\right)<\operatorname{dist}(x, y)<\delta$ it follows that $\left|\chi_{B}\left(\tau_{l_{0}} y_{0}\right)\right|>1 / 2 N$. So the transition from $y_{0}$ to $\tau_{l_{0}} y_{0}$. By induction, we can find $l_{1}, \ldots, l_{n}, \ldots$ such that the transitions from $\tau_{l_{n}} \ldots \tau_{l_{0}} x_{0}$ to $\tau_{l_{n+1}} \ldots \tau_{l_{0}} x_{0}$ and from $\tau_{l_{n}} \ldots \tau_{l_{0}} y_{0}$ to $\tau_{l_{n+1}} \ldots \tau_{l_{0}} y_{0}$ are possible. But

$$
\operatorname{dist}\left(\tau_{l_{n}} \ldots \tau_{l_{0}} x_{0}, \tau_{l_{n}} \ldots \tau_{l_{0}} y_{0}\right) \rightarrow 0 .
$$

Since $M_{1}, M_{2}$ are invariant this implies that $\operatorname{dist}\left(M_{1}, M_{2}\right)=0$, a contradiction.

Thus $\operatorname{dist}\left(M_{1}, M_{2}\right) \geq \delta$. Since all minimal closed $L$-invariant sets are contained in the compact set $X_{L}$, it follows that there are only finitely many of them.

Finally, we have to prove that the family of minimal sets is complete. For this take a closed invariant set $M$. Using Zorn's lemma, $M$ contains a minimal closed $L$-invariant set. This proves the completeness of the family.

Lemma 4.4. Let $M_{1}, M_{2}$ be two L-invariant sets with $\operatorname{dist}\left(M_{1}, M_{2}\right)>0$. Then for all $t_{1} \in M_{1}$ and $t_{2} \in M_{2}$ there exists $n \in \mathbb{N}$ such that for all words $w \in L^{n}$ of length $n$,

$$
\chi_{B}^{(n)}\left(\tau_{w} t_{1}\right) \chi_{B}^{(n)}\left(\tau_{w} t_{2}\right)=0 .
$$

Proof. Let $d_{0}:=\operatorname{dist}\left(M_{1}, M_{2}\right)$. We can assume that $\left(R^{T}\right)^{-1}$ is contractive under the metric dist. Pick $n$ such that $c^{n} \operatorname{dist}\left(t_{1}, t_{2}\right)<d_{0}$, where $0<c<1$ is a Lipschitz constant for all maps $\tau_{l}$, i.e.,

$$
\operatorname{dist}\left(\tau_{l} x, \tau_{l} y\right) \leq c \operatorname{dist}(x, y), \quad\left(x, y \in \mathbb{R}^{d}\right) .
$$

Consider a word $w=l_{1} \ldots l_{n}$ of length $n$. Suppose

$$
\chi_{B}^{(n)}\left(\tau_{w} t_{1}\right) \chi_{B}^{(n)}\left(\tau_{w} t_{2}\right) \neq 0 .
$$

Since $R$ and $L$ have integer entries, it follows that for $p=0, \ldots, n-1$,

$$
\chi_{B}\left(\left(R^{T}\right)^{p} \tau_{w} t_{1}\right)=\chi_{B}\left(\tau_{l_{n-p}} \ldots \tau_{l_{1}} t_{1}\right) .
$$

Then we have for $p=0, \ldots, n-1$

$$
\chi_{B}\left(\tau_{l_{n-p}} \ldots \tau_{l_{1}} t_{i}\right) \neq 0, \quad(i=1,2) .
$$

Since $M_{1}, M_{2}$ are invariant, this implies that $\tau_{w} t_{1} \in M_{1}, \tau_{w} t_{2} \in M_{2}$. But then

$$
d_{0} \leq \operatorname{dist}\left(\tau_{w} t_{1}, \tau_{w} t_{2}\right) \leq c^{n} \operatorname{dist}\left(t_{1}, t_{2}\right)<d_{0} .
$$

This contradiction implies our lemma.

Definition 4.5. Let $M$ be an $L$-invariant set. Define

$$
\begin{gathered}
\mathcal{K}_{M}:=\overline{\operatorname{span}}\left\{e_{-t}: t \in M\right\} \subset L^{2}\left(\mu_{B}\right), \\
\mathcal{H}(M):=\overline{\operatorname{span}}\left\{S_{w} e_{-t}: t \in M, w \in L^{n}, n \in \mathbb{N}\right\} .
\end{gathered}
$$

Theorem 4.6. Let $M$ be a closed L-invariant set.

(i) The space $\mathcal{K}_{M}$ is invariant under all the maps $S_{l}^{*}, l \in L$. 
(ii) The space $\mathcal{H}(M)$ is reducing for the representation $\left(S_{l}\right)_{l \in L}$ of the Cuntz algebra $\mathcal{O}_{N}$.

(iii) Let $M_{1}$ and $M_{2}$ be two closed L-invariant sets with $\operatorname{dist}\left(M_{1}, M_{2}\right)>0$. Then the subspaces $\mathcal{H}\left(M_{1}\right)$ and $\mathcal{H}\left(M_{2}\right)$ are orthogonal.

(iv) Let $\left(M_{i}\right)_{i=1}^{n}$ be a complete family of closed separated $L$-invariant sets. Then

$$
\bigoplus_{i=1}^{n} \mathcal{H}\left(M_{i}\right)=L^{2}\left(\mu_{B}\right)
$$

Proof. From Lemma 1.8, we have

$$
S_{l}^{*} e_{-t}=\chi_{B}\left(-\tau_{l}(t)\right) e_{-\tau_{l}(t)}=\overline{\chi_{B}\left(\tau_{l}(t)\right)} e_{-\tau_{l}(t)}
$$

for $t \in M$ and $l \in L$. So, if $\chi_{B}\left(\tau_{l}(t)\right)=0$, then $S_{l}^{*} e_{-t}=0$, otherwise, since $M$ is invariant $\tau_{l}(t) \in M$ so $S_{l}^{*} e_{-t} \in \mathcal{K}_{M}$. This proves (i).

(ii) follows from (i) and the Cuntz relations.

We now prove (iii). Iterating Lemma 1.8 we have for a word $w \in L^{n}$ and $t \in \mathbb{R}^{d}$ :

$$
S_{w}^{*} e_{-t}=\chi_{B}^{(n)}\left(-\tau_{w} t\right) e_{-\tau_{w} t} .
$$

First we prove that the subspaces $\mathcal{K}_{M_{1}}$ and $\mathcal{K}_{M_{2}}$ are orthogonal. Let $t_{1} \in M_{1}$ and $t_{2} \in M_{2}$. Take $n$ as in Lemma 4.4. For the $L^{2}\left(\mu_{B}\right)$-inner product we have:

$$
\begin{gathered}
\left\langle e_{-t_{1}}, e_{-t_{2}}\right\rangle=\left\langle e_{-t_{1}}, \sum_{w \in L^{n}} S_{w} S_{w}^{*} e_{-t_{2}}\right\rangle=\sum_{w \in L^{n}}\left\langle S_{w}^{*} e_{-t_{1}}, S_{w}^{*} e_{-t_{2}}\right\rangle \\
=\sum_{w \in L^{n}} \chi_{B}^{(n)}\left(-\tau_{w} t_{1}\right) \overline{\chi_{B}^{(n)}\left(-\tau_{w} t_{2}\right)}\left\langle e_{-\tau_{w} t_{1}}, e_{-\tau_{w} t_{2}}\right\rangle=0 .
\end{gathered}
$$

So the spaces $\mathcal{K}_{M_{1}}$ and $\mathcal{K}_{M_{2}}$ are orthogonal. Next, we prove that the spaces $\mathcal{H}\left(M_{1}\right)$ and $\mathcal{H}\left(M_{2}\right)$ are orthogonal. Take $w_{1}$ and $w_{2}$ to be two words over $L$. We can assume the length of $w_{1}$ is bigger than the length of $w_{2}$. Take $t_{1} \in M_{1}$ and $t_{2} \in M_{2}$.

We have from the Cuntz relations: $S_{w_{2}}^{*} S_{w_{1}}=0$ or $S_{w_{2}}^{*} S_{w_{1}}=S_{w}$ where $w$ is a subword of $w_{1}$. Only the second case requires some computations.

With (4.2) we have:

$$
\left\langle S_{w_{1}} e_{-t_{1}}, S_{w_{2}} e_{-t_{2}}\right\rangle=\left\langle S_{w}^{*} e_{-t_{1}}, e_{-t_{2}}\right\rangle=\chi_{B}^{(n)}\left(-\tau_{w} t_{1}\right)\left\langle e_{-\tau_{w} t_{1}}, e_{-t_{2}}\right\rangle .
$$

So, either $\chi_{B}^{(n)}\left(-\tau_{w} t_{1}\right) \neq 0$, in which case, since $M_{1}$ is invariant, $\tau_{w} t_{1} \in M_{1}$ so $e_{-\tau_{w} t_{1}}$ is in $\mathcal{K}_{M_{1}}$ and therefore the result of the previous computation is 0 , or, $\chi_{B}^{(n)}\left(-\tau_{w} t_{1}\right)=0$ in which case the result is again 0 .

Thus, (iii) follows.

We now prove (iv). Let $P$ be the projection onto the orthogonal sum of the subspaces $\mathcal{H}\left(M_{i}\right)$. Since the subspaces are reducing, we have that $P$ is in the commutant of the representation. With Corollary 1.13, the function

$$
h_{P}(t)=\left\langle P e_{-t}, e_{-t}\right\rangle_{L^{2}\left(\mu_{B}\right)}, \quad\left(t \in \mathbb{R}^{d}\right),
$$

is a fixed point of the transfer operator $R_{B, L}$. Assume $h_{P}$ is not identically 1 . We clearly have $0 \leq h_{P} \leq 1$.

Take $r>c \max _{l \in L}\|l\| /(1-c)$, where $\|\cdot\|$ is a norm under which the maps $\tau_{l}$ are contractive, and $c$ is the contractive constant. Then for the closed ball $\bar{B}(0, r)$, one 
has

$$
\bigcup_{l \in L} \tau_{l} \bar{B}(0, r) \subset \bar{B}(0, r)
$$

Let $m:=\min _{t \in \bar{B}(0, r)} h_{P}(t)$. Consider the set

$$
M:=\left\{t \in \bar{B}(0, r): h_{P}(t)=m\right\} .
$$

Take $t \in M$. We have

$$
m=h_{P}(t)=\left(R_{B, L} h_{P}\right)(t)=\sum_{l \in L}\left|\chi_{B}\left(\tau_{l} t\right)\right|^{2} h_{P}\left(\tau_{l} t\right) \geq m \sum_{l \in L}\left|\chi_{B}\left(\tau_{l} t\right)\right|^{2}=m,
$$

it follows that, if $\chi_{B}\left(\tau_{l} t\right) \neq 0$, then $h_{P}\left(\tau_{l} t\right)=m$. This shows that $M$ is a closed $L$-invariant set. But, since the family $\left(M_{i}\right)_{i}$ is complete, it follows that there is a $t \in M \cap M_{i}$ for some $i \in\{1, \ldots, n\}$.

But, if $t \in M_{i}$, then $e_{-t} \in \mathcal{H}\left(M_{i}\right)$ so $h_{P}(t)=1$. On the other hand, $t \in M$ so $h_{P}(t)=m$. So $m=1$. Therefore $h_{P}$ is constant 1 on $\bar{B}(0, r)$. For $r \rightarrow \infty$, we get that $h_{P}=1$ on $\mathbb{R}^{d}$. This implies that $e_{-t}$ is in the range of $P$ for all $t \in \mathbb{R}^{d}$. But, by the Stone-Weierstrass theorem $e_{-t}, t \in \mathbb{R}^{d}$ span the entire space $L^{2}\left(\mu_{B}\right)$. So (iv) follows.

Example 4.7. Consider the following data:

$$
R=\left[\begin{array}{ll}
2 & 0 \\
1 & 2
\end{array}\right], \quad B=\left\{\left(\begin{array}{l}
0 \\
0
\end{array}\right),\left(\begin{array}{l}
1 \\
0
\end{array}\right),\left(\begin{array}{l}
0 \\
p
\end{array}\right),\left(\begin{array}{l}
1 \\
p
\end{array}\right)\right\} \quad L=\left\{\left(\begin{array}{l}
0 \\
0
\end{array}\right),\left(\begin{array}{l}
1 \\
0
\end{array}\right),\left(\begin{array}{l}
0 \\
1
\end{array}\right),\left(\begin{array}{l}
1 \\
1
\end{array}\right)\right\}
$$

where $p \in \mathbb{N}$ is an odd number.
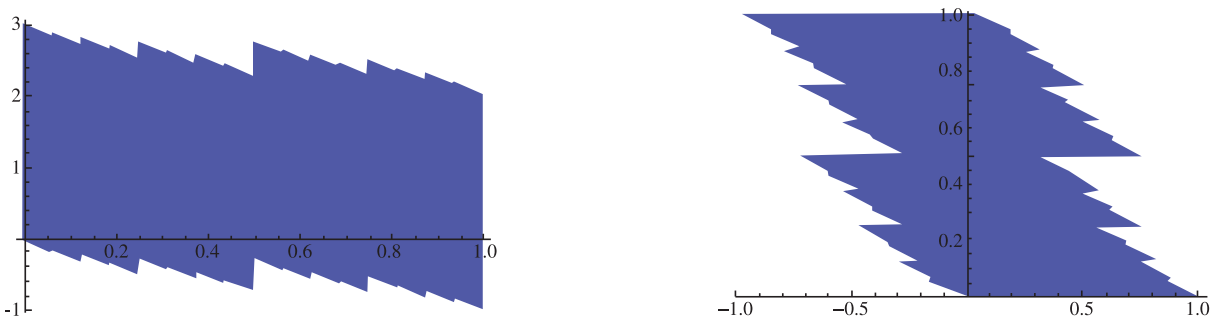

Figure 1. The attractors $X(B)$ and $X(L)$ for $p=3$

We will prove first that the invariant measure $\mu_{B}$ is the Lebesgue measure on the attractor $X_{B}$, renormalized so that the total measure is 1 .

First, define the function $g:[0,1] \rightarrow \mathbb{R}$, for $x=\sum_{n=1}^{\infty} x_{n} / 2^{n}$, with $x_{n} \in\{0,1\}$ for all $n$,

$$
g(x)=-\sum_{n=1}^{\infty} \frac{n}{2} \frac{x_{n}}{2^{n}}
$$

The number $g(x)$ will represent the lowest endpoint of the vertical slice of $X_{B}$ at $x$. We claim that $g$ has the following property:

$$
g\left(\frac{x+b}{2}\right)=-\frac{x+b}{4}+\frac{g(x)}{2}, \quad(x \in[0,1], b \in\{0,1\})
$$


Indeed, if $x=\sum_{n=1}^{\infty} x_{n} / 2^{n}$, then $(x+b) / 2=b / 2+x_{1} / 2^{2}+\cdots+x_{n} / 2^{n+1}+\ldots$, so

$$
\begin{aligned}
g\left(\frac{x+b}{2}\right) & =-\left(-\frac{1}{2} \frac{b}{2}+\frac{2}{2} \frac{x_{1}}{2^{2}}+\cdots+\frac{n+1}{2} \frac{x_{n}}{2^{n+1}}+\ldots\right) \\
& =-\left(\frac{b}{4}+\frac{1}{4}\left(\frac{x_{1}}{2}+\cdots+\frac{x_{n}}{2^{n}}+\ldots\right)\right)+\frac{1}{2}\left(\frac{1}{2} \frac{x_{1}}{2}+\cdots+\frac{n}{2} \frac{x_{n}}{2^{n}}+\ldots\right) \\
& =-\frac{x+b}{4}+\frac{g(x)}{2} .
\end{aligned}
$$

We prove now that the measure $\mu_{B}$ is given (up to the renormalization factor $1 / p)$ by

$$
\int_{0}^{1} \int_{0}^{p} f(x, g(x)+y) d y d x
$$

For this, we have to check the invariance equation. We have

$$
\begin{aligned}
\frac{1}{4} & \sum_{b_{1} \in\{0,1\}} \sum_{b_{2} \in\{0, p\}} \int_{0}^{1} \int_{0}^{p} f \circ \tau_{\left(b_{1}, b_{2}\right)^{T}}(x, g(x)+y) d y d x \\
= & \frac{1}{4} \sum_{b_{1} \in\{0,1\}} \sum_{b_{2} \in\{0, p\}} \int_{0}^{1} \int_{0}^{p} f\left(\frac{x+b_{1}}{2},-\frac{x+b_{1}}{4}+\frac{g(x)+y+b_{2}}{2}\right) d y d x \\
= & \frac{1}{4} \sum_{b_{1} \in\{0,1\}} \sum_{b_{2} \in\{0, p\}} \int_{0}^{1} \int_{0}^{p} f\left(\frac{x+b_{1}}{2}, g\left(\frac{x+b_{1}}{2}\right)+\frac{y+b_{2}}{2}\right) d y d x \\
= & \sum_{b_{1} \in\{0,1\}} \sum_{b_{2} \in\{0, p\}} \int_{\frac{b_{1}}{2}}^{\frac{b_{1}+1}{2}} \int_{\frac{b_{2}}{2}}^{\frac{b_{2}+p}{2}} f(x, g(x)+y) d y d x \\
= & \int_{0}^{1} \int_{0}^{p} f(x, g(x)+y) d y d x .
\end{aligned}
$$

Lemma 4.8. The function $g$ is continuous at points $x$ which are dyadic-irrational (i.e., not of the form $x=k / 2^{n}$ ), and it has jump discontinuities at dyadic rational values $x$. The sum of the jumps at the discontinuities is infinite.

Proof. Set $x=1 / 2$, then since $1 / 2$ has two binary expansions $.0111 \ldots$ and $.1000 \ldots$, we get that

$$
g(1 / 2)=-1 / 4 \text { and } g(1 / 2)_{-}=-\sum_{n=2}^{\infty} \frac{n}{2^{n+1}}=-\frac{3}{4} .
$$

Therefore the jump at $x$ is $D(1 / 2)=1 / 2$. Using the relation (4.3), and induction we get that the jump at $k / 2^{n}$ with $k$ odd, $k<2^{n}$ and $n \geq 1$ is $D\left(k / 2^{n}\right)=1 / 2^{n+1}$.

Since there are $2^{n-1}$ distinct dyadics of the form $k / 2^{n}$ with $0<k<2^{n}, k$ odd, the sum of the jumps is at least $\sum_{n=1}^{\infty} 2^{n-1} \frac{1}{2^{n}}=\infty$.

Since the binary representation is unique when $x$ is dyadic-irrational, it follows that $g$ is continuous at such points.

Lemma 4.9. The function $g$ is nowhere differentiable.

Proof. From the proof of Lemma 4.8 we see that each interval of length $\frac{1}{2^{n}}$ contains a dyadic of the form $k / 2^{n}$ so it contains a pair of points $x, y$ such that $|g(x)-g(y)|>$ 
$\frac{1}{2^{n+2}}$. Then

$$
\left|\frac{g(x)-g(y)}{x-y}\right|>\frac{1}{4}
$$

Lemma 4.10. The two attractors $X(B)$ and $X(L)$ are given by (see Figure 1$)$

$$
\begin{aligned}
& X(B)=\left\{(x, y) \in \mathbb{R}^{2}: 0 \leq x \leq 1, g(x) \leq y \leq g(x)+p\right\}, \\
& X(L)=\left\{(\xi, \eta) \in \mathbb{R}^{2}: 0 \leq \eta \leq 1, g(\eta) \leq \xi \leq g(\eta)+1\right\} .
\end{aligned}
$$

Proof. The result follows from (4.4) and the fact that $X(B)$ is the support of $\mu_{B}$. For $X(L)$ the proof is analogous.

The computation for (4.4) proves that

Lemma 4.11. The measure $\mu_{B}$ is the $\frac{1}{p}$ times the Lebesgue measure restricted to $X(B)$.

Lemma 4.12. The Fourier transform of the measure $\mu_{B}$ is

$$
\hat{\mu}_{B}\left(t_{1}, t_{2}\right)=\int_{0}^{1} e^{2 \pi i\left(t_{1} x+t_{2} g(x)\right)} d x \cdot \frac{e^{2 \pi i p t_{2}}-1}{2 \pi i p t_{2}}
$$

for $\left(t_{1}, t_{2}\right) \in \mathbb{R}^{2}$.

Proof. Immediate from (4.4).

Lemma 4.13. Let $\mu_{B}$ as above. Then

$$
\sum_{n \in \mathbb{Z}}\left|\hat{\mu}_{B}\left(t_{1}+n, t_{2}\right)\right|^{2}=\frac{\sin ^{2}\left(p \pi t_{2}\right)}{\left(p \pi t_{2}\right)^{2}}, \quad\left(\left(t_{1}, t_{2}\right) \in \mathbb{R}^{2}\right)
$$

Proof. Using Lemma 4.12 we get

$$
\begin{gathered}
\sum_{n \in \mathbb{Z}}\left|\hat{\mu}_{B}\left(t_{1}+n, t_{2}\right)\right|^{2}=\sum_{n \in \mathbb{Z}}\left|\int_{0}^{1} e_{n}(x) e^{2 \pi i\left(t_{1} x+t_{2} g(x)\right)} d x\right|^{2} \frac{\sin ^{2}\left(p \pi t_{2}\right)}{\left(p \pi t_{2}\right)^{2}} \\
=(\text { using Parseval's relation })=\frac{\sin ^{2}\left(p \pi t_{2}\right)}{\left(p \pi t_{2}\right)^{2}} .
\end{gathered}
$$

Proposition 4.14. The set $\mathbb{Z} \times \frac{1}{p} \mathbb{Z}$ is a spectrum for the measure $\mu_{B}$. The set $X(B)$ tiles $\mathbb{R}^{2}$ by $\mathbb{Z} \times p \mathbb{Z}$.

Proof. We have to prove that

$$
\sum_{\gamma \in \mathbb{Z} \times \frac{1}{p} \mathbb{Z}}\left|\hat{\mu}_{B}(t+\gamma)\right|^{2}=1, \quad\left(t \in \mathbb{R}^{2}\right)
$$

(see e.g. DJ06b for details).

$$
\begin{aligned}
& \sum_{\gamma \in \mathbb{Z} \times \frac{1}{p} \mathbb{Z}}\left|\hat{\mu}_{B}(t+\gamma)\right|^{2}=\sin ^{2}\left(p \pi t_{2}\right) \sum_{k \in \mathbb{Z}} \frac{1}{\left(p \pi\left(t_{2}+\frac{k}{p}\right)\right)^{2}} \\
& =\sin ^{2}\left(p \pi t_{2}\right) \sum_{k \in \mathbb{Z}} \frac{1}{\pi^{2}\left(p t_{2}+k\right)^{2}}=\frac{\sin ^{2}\left(p \pi t_{2}\right)}{\sin ^{2}\left(p \pi t_{2}\right)}=1 .
\end{aligned}
$$


Proposition 4.15. The B-extreme L-cycles are

$$
\left\{\left(\begin{array}{l}
0 \\
0
\end{array}\right)\right\},\left\{\left(\begin{array}{l}
1 \\
0
\end{array}\right)\right\},\left\{\left(\begin{array}{l}
0 \\
1
\end{array}\right)\right\},\left\{\left(\begin{array}{c}
-1 \\
1
\end{array}\right)\right\}
$$

and the associated words are, respectively,

$$
\left(\begin{array}{l}
0 \\
0
\end{array}\right),\left(\begin{array}{l}
1 \\
0
\end{array}\right),\left(\begin{array}{l}
1 \\
1
\end{array}\right),\left(\begin{array}{l}
0 \\
1
\end{array}\right) \text {. }
$$

Proof. For this we can use Theorem 3.2. Note that $\Gamma(B)^{\circ}=\mathbb{Z} \times \mathbb{Z}$.

Remark 4.16. For later use, we record the following points on the graph of $g$ :

$$
g\left(\frac{1}{3}\right)=-\frac{4}{9}, \quad g\left(\frac{2}{3}\right)=-\frac{5}{9} .
$$

To see this, set $x=g(1 / 3)$ and $y=g(2 / 3)$. Use (4.3): clearly, $\frac{\frac{1}{3}+1}{2}=\frac{2}{3}$ and $\frac{\frac{2}{3}+0}{2}=\frac{1}{3}$ :

$$
\begin{aligned}
& x=g\left(\frac{\frac{2}{3}+0}{2}\right)=-\frac{\frac{2}{3}+0}{4}+\frac{y}{2}, \\
& y=g\left(\frac{\frac{1}{3}+1}{2}\right)=-\frac{\frac{1}{3}+1}{4}+\frac{x}{2},
\end{aligned}
$$

and then solve for $x$ and $y$.

The numbers in (4.8) result from intersecting the respective (horizontal) line segments $y=1 / 3$ and $y=2 / 3$ with the compact tile $X(L)$ in Figure 1 . The invariant set $M$ (Definition 4.2) is the union of these two segments; see also (4.6) in Lemma 4.10, Each line segment is unit-length, and the numbers in (4.8) are the respective left-hand side endpoints.

The infinite random walk defined by restricting the maps $\tau_{l}$ to $M$ (Proposition 4.3) turns out in Example 4.7 to be a zigzag motion, with the walker making successive jumps back and forth between the two line-segments.

In the discussion below, we find the cycles $C$ from section 3 , and the corresponding orthogonal sets $\Lambda(C)$, (illustrated in Figure 2). Conclusion: Example 4.7 has one noncycle invariant set $M$, and 4 distinct cycles $C$.

Remark 4.17. We want to describe the sets $\Lambda(C)$ corresponding to the cycles in Proposition 4.15] We need some preliminaries. Each nonnegative integer $k \geq 0$ can be represented in base 2 as

$$
k=j_{0}+2 j_{1}+2^{2} j_{2}+\cdots+2^{n} j_{n} .
$$

The representation is unique, up to adding 0 digits at the end. Each negative integer $k<0$ can be represented as

$$
k=-2^{n+1}+j_{0}+2 j_{1}+2^{2} j_{2}+\cdots+2^{n} j_{n} .
$$

The representation is unique up to adding 1 digits at the end.

We define the following function:

$$
\text { For } k \geq 0, k=j_{0}+2 j_{1}+\cdots+2^{n} j_{n}, \quad h(k):=j_{1}+2 \cdot 2^{1} j_{2}+\cdots+n 2^{n-1} j_{n},
$$

(4.10) For $k<0, k=-2^{n+1}+j_{0}+2 j_{1}+\cdots+2^{n} j_{n}$,

$$
h(k):=2^{n+1}-(n+1) 2^{n}+j_{1}+2 \cdot 2^{1} j_{2}+\cdots+n 2^{n-1} j_{n} .
$$


It is easy to check that the function $h$ is well defined, i.e., it does not depend on the representation, and it satisfies the following equation

$$
2 h(n)+n=h\left(2 n+j_{0}\right), \text { for all } n \in \mathbb{Z}, j_{0} \in\{0,1\} .
$$

Proposition 4.18. The four sets $\Lambda(C)$ (as in Theorem 3.4) for the four cycles in Proposition 4.15] are

$$
\begin{aligned}
& \Lambda\left(\left\{\left(\begin{array}{l}
0 \\
0
\end{array}\right)\right\}\right)=\left\{\left(\begin{array}{l}
t_{1} \\
t_{2}
\end{array}\right) \in \mathbb{Z}^{2}: t_{2} \geq 0, t_{1} \geq h\left(t_{2}\right)\right\}, \\
& \Lambda\left(\left\{\left(\begin{array}{c}
-1 \\
1
\end{array}\right)\right\}\right)=\left\{\left(\begin{array}{c}
t_{1} \\
t_{2}
\end{array}\right) \in \mathbb{Z}^{2}: t_{2}<0, t_{1} \geq h\left(t_{2}\right)\right\}, \\
& \Lambda\left(\left\{\left(\begin{array}{l}
0 \\
1
\end{array}\right)\right\}\right)=\left\{\left(\begin{array}{l}
t_{1} \\
t_{2}
\end{array}\right) \in \mathbb{Z}^{2}: t_{2}<0, t_{1}<h\left(t_{2}\right)\right\}, \\
& \Lambda\left(\left\{\left(\begin{array}{l}
1 \\
0
\end{array}\right)\right\}\right)=\left\{\left(\begin{array}{l}
t_{1} \\
t_{2}
\end{array}\right) \in \mathbb{Z}^{2}: t_{2} \geq 0, t_{1}<h\left(t_{2}\right)\right\},
\end{aligned}
$$

and as a result

$$
\bigcup_{C} \Lambda(C)=\mathbb{Z}^{2}, \quad \Lambda(0,0) \cup \Lambda(1,0)=\mathbb{Z} \times \mathbb{Z}_{\geq 0}
$$

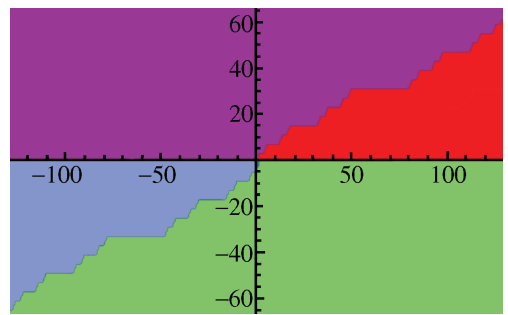

Figure 2. The sets $\Lambda(C)$

Proof. Let $A=R^{T}$. Take a point $(k, l) \in \mathbb{Z}^{2}$. Then, since $L$ is a complete set of representatives for $\mathbb{Z}^{2} / A \mathbb{Z}^{2}$, it follows that there is a unique $(m, n) \in \mathbb{Z}^{2}$ and $\left(l_{0}, l_{1}\right) \in L$ such that $(k, l)^{T}=A(m, n)^{T}+\left(l_{0}, l_{1}\right)^{T}$. We consider the map $r$ : $(k, l) \mapsto(m, n)$. Using (4.11), a computation shows that the four sets $\Lambda(C)$ are invariant under this map. Iterating the map $r$, each point is eventually mapped into $-C$ for one of the cycles $C$. For more details check also BJ99, DJP09.

Remark 4.19. The sum of the subspaces $\mathcal{H}(C)$ (as in Theorem 3.4) where $C$ runs over the $B$-extreme $L$-cycles is not total in $L^{2}\left(\mu_{B}\right)$. This is because the spectrum of $\mu_{B}$ is $\mathbb{Z} \times \frac{1}{p} \mathbb{Z}$, not $\mathbb{Z} \times \mathbb{Z}$. Actually, all the exponential functions $e_{(t, l / p)^{T}}, l \not \equiv 0 \bmod p$ are orthogonal to this sum. Indeed, for $\left(n_{1}, n_{2}\right) \in \mathbb{Z}^{2}$ :

$$
\left\langle e_{(t, l / p)^{T}}, e_{\left(n_{1}, n_{2}\right)^{T}}\right\rangle_{L^{2}\left(\mu_{B}\right)}=\int_{0}^{1} e^{2 \pi i\left(\left(t-n_{1}\right) x+\left(\frac{l}{p}-n_{2}\right) g(x)\right)} \int_{0}^{p} e^{2 \pi i\left(\frac{l}{p}-n_{2}\right) y} d y d x=0 .
$$

Lemma 4.20. Let $(R, B, L)$ be a Hadamard system in $\mathbb{R}^{d}$, and let $\Gamma \subset \mathbb{R}^{d}$ be a subset such that the vectors $\left\{e_{\gamma}: \gamma \in \Gamma\right\}$ are orthogonal $L^{2}\left(\mu_{B}\right)$. Let $P_{\Gamma}$ be the 
projection onto the corresponding subspace in $L^{2}\left(\mu_{B}\right)$ spanned by these vectors, and let

$$
h_{P_{\Gamma}}(t):=\left\|P_{\Gamma} e_{-t}\right\|^{2}, \quad\left(t \in \mathbb{R}^{d}\right) .
$$

Then

$$
h_{P_{\Gamma}}(t)=\sum_{\gamma \in \Gamma}\left|\hat{\mu}_{B}(t+\gamma)\right|^{2}, \quad\left(t \in \mathbb{R}^{d}\right)
$$

and $0 \leq h_{P_{\Gamma}}(t) \leq 1$ for all $t \in \mathbb{R}^{d}$.

Proof. Since the vectors are orthogonal we have

$$
P_{\Gamma}=\sum_{\gamma \in \Gamma}\left|e_{\gamma}\right\rangle\left\langle e_{\gamma}\right|
$$

with the use of Dirac notation for rank-1 operators. Since

$$
\|\left|e_{\gamma}\right\rangle\left\langle\left. e_{\gamma}\left|e_{-t} \|^{2}=\right| \hat{\mu}_{B}(t+\gamma)\right|^{2},\right.
$$

a substitution into (4.12) yields the desired conclusions.

Corollary 4.21. For Example 4.7, corresponding to the four cycles in Proposition 4.18, we get the four independent harmonic functions

$$
h_{C}(t)=\left\|P_{\Lambda(C)} e_{-t}\right\|^{2}, \quad\left(t \in \mathbb{R}^{2}\right)
$$

and

$$
\sum_{C} h_{C}(t)=\left\|P_{\mathbb{Z}^{2}} e_{-t}\right\|^{2}=\left(\frac{\sin p \pi t_{2}}{p \sin \pi t_{2}}\right)^{2}, \quad\left(\left(t_{1}, t_{2}\right) \in \mathbb{R}^{2}\right) .
$$

Proof. It follows from Proposition 4.18 that the sum of the four harmonic functions in (4.14) is $\left\|P_{\mathbb{Z}^{2}} e_{-t}\right\|^{2}$. An application of Lemma 4.20 and 4.13 then yields

$$
\left\|P_{\mathbb{Z}^{2}} e_{-t}\right\|^{2}=\frac{\sin ^{2}\left(p \pi t_{2}\right)}{(p \pi)^{2}} \sum_{n \in \mathbb{Z}} \frac{1}{(t+n)^{2}}=\frac{\sin ^{2}\left(p \pi t_{2}\right)}{p^{2} \sin ^{2}\left(\pi t_{2}\right)}
$$

which is the desired conclusion (4.14).

Remark 4.22. The functions

$$
f_{A}(t)=\left\langle A e_{-t}, e_{-t}\right\rangle_{L^{2}\left(\mu_{B}\right)}
$$

in Lemma 4.20 are entirely analytic on $\mathbb{R}^{d}$ for every bounded operator $A$. In fact, the functions in Corollary 4.21 are trigonometric polynomials. Indeed, the expression on the right-hand side in (4.14) divides, for example, for $p=3$, we have

$$
\frac{\sin 3 \pi t}{\sin \pi t}=3-4 \sin ^{2}(\pi t)=4 \cos ^{2}(\pi t)-1
$$

Remark 4.23. In affine examples, it is typically more difficult to obtain explicit formulas for the individual harmonic functions $h_{C}(t)$ (as in Proposition 4.18) than for their summations. The difference may be explained by the difference between number theoretic expressions such as

$$
\sum_{n \in \mathbb{Z}} \frac{1}{(t+n)^{2}}=\frac{\pi^{2}}{\sin ^{2}(\pi t)}
$$


on the one hand, and

$$
\zeta_{2}(t)=\sum_{n=0}^{\infty} \frac{1}{(t+n)^{2}}=\left(\frac{d}{d t}\right)^{2} \log \Gamma(t),
$$

i.e., summation over $\mathbb{Z}_{\geq 0}$, where $\Gamma$ is the usual Gamma function. The function $\zeta_{2}$ in (4.16) is one of the Hurwitz-Riemann zeta functions [AAR99.

Using this, we arrive at the following:

$$
h_{\Lambda(0,0)}(t)+h_{\Lambda(1,0)}(t)=\frac{\sin ^{2}\left(p \pi t_{2}\right)}{\pi^{2}} \zeta_{2}\left(t_{2}\right)
$$

and

$$
h_{\Lambda(-1,1)}(t)+h_{\Lambda(0,1)}(t)=\frac{\sin ^{2}\left(p \pi t_{2}\right)}{\pi^{2}}\left(-\frac{1}{t_{2}^{2}}+\zeta_{2}\left(-t_{2}\right)\right)
$$

for all $t=\left(t_{1}, t_{2}\right) \in \mathbb{R}^{2}$. In all cases the functions $t \mapsto h_{\Lambda(C)}(t)$ have entire analytic extensions.

Proposition 4.24. Fi $p \in \mathbb{Z}$ odd, and let $(R, B, L)$ be as in Example 4.7. List the nontrivial orbits $O_{1}, O_{2}, \ldots$ for $x \mapsto 2 x$ acting on $\mathbb{Z}_{p}=\mathbb{Z} / p \mathbb{Z}=\{0,1,2, \ldots, p-1\}$. Then the sets

$$
M\left(O_{i}\right)=X(L) \cap\left\{\left(\begin{array}{l}
\xi \\
\eta
\end{array}\right): p \eta \in O_{i}\right\}
$$

are minimal invariant sets

Remark 4.25. If $p=3$, there is only one orbit $O_{1}=\{1,2\}$. For $p=5: O_{1}=$ $\{1,2,4,3\}$. If $p=7$, the list of nontrivial orbits is $O_{1}=\{1,2,4\}$, and $O_{2}=\{3,6,5\}$. If $p=9$ the list is $O_{1}=\{1,2,4,8,7,5\}, O_{2}=\{3,6\}$.

For general values of $p$, the orbits can be computed, and the number of possible orbits gets arbitrarily large.

Proof of Proposition 4.24. This is based on the formula (4.2) in the proof of Theorem 4.6 above. When applied to Example 4.7, we get

$$
\tau_{\left(l_{1}, l_{2}\right)}^{(L)}\left(t_{1}, t_{2}\right)=\left(\begin{array}{c}
\frac{1}{2}\left(t_{1}+l_{1}-\frac{t_{2}+l_{2}}{2}\right) \\
\frac{t_{2}+l_{2}}{2}
\end{array}\right) .
$$

Then

$$
\left|\chi_{B}\left(\tau_{\left(l_{1}, l_{2}\right)}^{(L)}\left(t_{1}, t_{2}\right)\right)\right|^{2}=\cos ^{2}\left(\pi \frac{1}{2}\left(t_{1}+l_{1}-\frac{t_{2}+l_{2}}{2}\right) \cos ^{2}\left(\frac{p \pi}{2}\left(t_{2}+l_{2}\right)\right),\right.
$$

and the conclusion follows.

The details for $p=3$ are as follows $(l \in\{0,1\})$ :

$$
\left|\chi_{B}\left(\tau_{(l, 1)}^{(L)}(t, 1 / 3)\right)\right|^{2}=\cos ^{2}\left(\frac{\pi}{2}\left(t+l-\frac{2}{3}\right)\right)
$$

while

$$
\left|\chi_{B}\left(\tau_{(l, 0)}^{(L)}(t, 1 / 3)\right)\right|^{2}=0
$$

The transition probabilities in the other direction are

and

$$
\left|\chi_{B}\left(\tau_{(l, 0)}^{(L)}(t, 2 / 3)\right)\right|^{2}=\cos ^{2}\left(\frac{\pi}{2}\left(t+l-\frac{1}{3}\right)\right)
$$

$$
\left|\chi_{B}\left(\tau_{(l, 1)}^{(L)}(t, 2 / 3)\right)\right|^{2}=0
$$


With the use of (4.18) and (4.19) we get the remaining conclusion of the proposition.

Corollary 4.26. Let $p=3$ in Example 4.7. Then there is just one noncycle minimal invariant set

$M=X(L) \cap\left\{\left(\begin{array}{l}\xi \\ \eta\end{array}\right): \eta \in\left\{\frac{1}{3}, \frac{2}{3}\right\}\right\}=\left\{\left(\begin{array}{c}t \\ \frac{1}{3}\end{array}\right):-\frac{4}{9} \leq t \leq \frac{5}{9}\right\} \cup\left\{\left(\begin{array}{c}t \\ \frac{2}{3}\end{array}\right):-\frac{5}{9} \leq t \leq \frac{4}{9}\right\}$.

If $Q$ is the projection onto $\mathcal{H}(M) \subset L^{2}\left(\mu_{B}\right)$, then the associated harmonic function is

$$
h_{Q}(t)=1-\left(1-\frac{4}{3} \sin ^{2}\left(\pi t_{2}\right)\right)^{2}, \quad\left(t=\left(t_{1}, t_{2}\right) \in \mathbb{R}^{2}\right)
$$

Proof. Combine the results in Lemma 4.20, Proposition 4.24 and Remark 4.16,

\section{REFERENCES}

[AAR99] George E. Andrews, Richard Askey, and Ranjan Roy. Special functions, volume 71 of Encyclopedia of Mathematics and its Applications. Cambridge University Press, Cambridge, 1999. MR1688958 (2000g:33001)

[ACHM07] Akram Aldroubi, Carlos Cabrelli, Douglas Hardin, and Ursula Molter. Optimal shift invariant spaces and their Parseval frame generators. Appl. Comput. Harmon. Anal., 23(2):273-283, 2007. MR2344616(2008i:94010)

[AdLM02] K. T. Arasu, Warwick de Launey, and S. L. Ma. On circulant complex Hadamard matrices. Des. Codes Cryptogr., 25(2):123-142, 2002. MR1883962 (2003b:05036)

[Bar09] Michael F. Barnsley. Transformations between self-referential sets. Amer. Math. Monthly, 116(4):291-304, 2009. MR2503315 (2010b:28009)

[BHS05] Michael Barnsley, John Hutchinson, and Orjan Stenflo. A fractal valued random iteration algorithm and fractal hierarchy. Fractals, 13(2):111-146, 2005. MR.2151094 (2006b:28014)

[BJ97a] O. Bratteli and P. E. T. Jorgensen. Endomorphisms of $\mathcal{B}(\mathcal{H})$. II. Finitely correlated states on $\mathcal{O}_{n}$. J. Funct. Anal., 145(2):323-373, 1997. MR.1444086 (98c:46128)

[BJ97b] Ola Bratteli and Palle E. T. Jorgensen. Isometries, shifts, Cuntz algebras and multiresolution wavelet analysis of scale $N$. Integral Equations Operator Theory, 28(4):382-443, 1997. MR1465320 (99k:46094b)

[BJ99] Ola Bratteli and Palle E. T. Jorgensen. Iterated function systems and permutation representations of the Cuntz algebra. Mem. Amer. Math. Soc., 139(663):x+89, 1999. MR 1469149 (99k:46094a)

[BJKW00] O. Bratteli, P. E. T. Jorgensen, A. Kishimoto, and R. F. Werner. Pure states on $\mathcal{O}_{d}$. J. Operator Theory, 43(1):97-143, 2000. MR.1740897(2001b:46086)

[BL07] William D. Banks and Florian Luca. Sums of prime divisors and Mersenne numbers. Houston J. Math., 33(2):403-413 (electronic), 2007. MR2308986 (2008d:11112)

[BV05] Joseph A. Ball and Victor Vinnikov. Functional models for representations of the Cuntz algebra. In Operator theory, systems theory and scattering theory: multidimensional generalizations, volume 157 of Oper. Theory Adv. Appl., pages 1-60. Birkhäuser, Basel, 2005. MR2129642(2006e:47023)

[CCR96] D. Cerveau, J.-P. Conze, and A. Raugi. Ensembles invariants pour un opérateur de transfert dans $\mathbf{R}^{d}$. Bol. Soc. Brasil. Mat. (N.S.), 27(2):161-186, 1996. MR1418931 (98m:28044)

[CHK97] R. Craigen, W. H. Holzmann, and H. Kharaghani. On the asymptotic existence of complex Hadamard matrices. J. Combin. Des., 5(5):319-327, 1997. MR.1465343 (99e:05026)

[CHR97] J.-P. Conze, L. Hervé, and A. Raugi. Pavages auto-affines, opérateurs de transfert et critères de réseau dans $\mathbf{R}^{d}$. Bol. Soc. Brasil. Mat. (N.S.), 28(1):1-42, 1997. MR.1444447(99a:52034) 
[CM07] Alain Connes and Matilde Marcolli. Renormalization, the Riemann-Hilbert correspondence, and motivic Galois theory. In Frontiers in number theory, physics, and geometry. II, pages 617-713. Springer, Berlin, 2007. MR2290770 (2008g:81156)

[CR90] Jean-Pierre Conze and Albert Raugi. Fonctions harmoniques pour un opérateur de transition et applications. Bull. Soc. Math. France, 118(3):273-310, 1990. MR.1078079 (92g:60100)

[Cun77] Joachim Cuntz. Simple $C^{*}$-algebras generated by isometries. Comm. Math. Phys., 57(2):173-185, 1977. MR0467330 (57:7189)

[Den09] Qi-Rong Deng. Reverse iterated function system and dimension of discrete fractals. Bull. Aust. Math. Soc., 79(1):37-47, 2009. MR2486879 (2010j:28013)

[DFdGtHR04] Remco Duits, Luc Florack, Jan de Graaf, and Bart ter Haar Romeny. On the axioms of scale space theory. J. Math. Imaging Vision, 20(3):267-298, 2004. MR2060148(2005k:94005)

[Dit04] P. Dita. Some results on the parametrization of complex Hadamard matrices. $J$. Phys. A, 37(20):5355-5374, 2004. MR2065675 (2005b:15045)

[DJ06a] Dorin E. Dutkay and Palle E. T. Jorgensen. Wavelets on fractals. Rev. Mat. Iberoam., 22(1):131-180, 2006. MR2268116 (2008h:42071)

[DJ06b] Dorin Ervin Dutkay and Palle E. T. Jorgensen. Iterated function systems, Ruelle operators, and invariant projective measures. Math. Comp., 75(256):1931-1970 (electronic), 2006. MR2240643 (2008h:28005)

[DJ07] Dorin Ervin Dutkay and Palle E. T. Jorgensen. Analysis of orthogonality and of orbits in affine iterated function systems. Math. Z., 256(4):801-823, 2007. MR2308892 (2009e:42013)

[DJP09] Dorin Ervin Dutkay, Palle E.T. Jorgensen, and Gabriel Picioroaga. Unitary representations of wavelet groups and encoding of iterated function systems in solenoids. Erg. Th. Dyn. Sys., 29(6):1815-1852, 2009. MR2563094(2011e:22009)

[DLC09] Xiaoyan Deng, Helong Li, and Xiaokun Chen. The symbol series expression and Hölder exponent estimates of fractal interpolation function. J. Comput. Anal. Appl., 11(3):507-523, 2009. MR2530679(2010i:28010)

[FLS09] Kevin Ford, Florian Luca, and Igor E. Shparlinski. On the largest prime factor of the Mersenne numbers. Bull. Aust. Math. Soc., 79(3):455-463, 2009. MR2505350 (2010b:11126)

[Fug74] Bent Fuglede. Commuting self-adjoint partial differential operators and a group theoretic problem. J. Functional Analysis, 16:101-121, 1974. MR0470754 (57:10500)

[GR09] Daniel Goncalves and Danilo Royer. Perron-Frobenius operators and representations of the Cuntz-Krieger algebras for infinite matrices. J. Math. Anal. Appl., 351(2):811-818, 2009. MR2473986 (2010b:46120)

[HL08a] Xing-Gang He and Ka-Sing Lau. On a generalized dimension of self-affine fractals. Math. Nachr., 281(8):1142-1158, 2008. MR2427166 (2010a:28009)

[HL08b] Tian-You Hu and Ka-Sing Lau. Spectral property of the Bernoulli convolutions. Adv. Math., 219(2):554-567, 2008. MR2435649 (2010a:42094)

[Hut81] John E. Hutchinson. Fractals and self-similarity. Indiana Univ. Math. J., 30(5):713-747, 1981. MR625600 (82h:49026)

[Jør82] Palle E. T. Jørgensen. Spectral theory of finite volume domains in $\mathbf{R}^{n}$. Adv. in Math., 44(2):105-120, 1982. MR658536 (84k:47024)

[Jor06] Palle E. T. Jorgensen. Analysis and probability: wavelets, signals, fractals, volume 234 of Graduate Texts in Mathematics. Springer, New York, 2006. MR2254502 (2008a:42030)

[KS93] H. Kharaghani and Jennifer Seberry. The excess of complex Hadamard matrices. Graphs Combin., 9(1):47-56, 1993. MR1215584 (94f:05028)

[LL07] King-Shun Leung and Ka-Sing Lau. Disklikeness of planar self-affine tiles. Trans. Amer. Math. Soc., 359(7):3337-3355 (electronic), 2007. MR:2299458 (2008k:52046)

[MM09] Alexandru Mihail and Radu Miculescu. The shift space for an infinite iterated function system. Math. Rep. (Bucur.), 11(61)(1):21-32, 2009. MR2506506 (2010b:28022) 
[MP04] Leo Murata and Carl Pomerance. On the largest prime factor of a Mersenne number. In Number theory, volume 36 of CRM Proc. Lecture Notes, pages 209-218. Amer. Math. Soc., Providence, RI, 2004. MR2076597 (2005i:11137)

[MZ09] Ursula M. Molter and Leandro Zuberman. A fractal Plancherel theorem. Real Anal. Exchange, 34(1):69-85, 2009. MR 2527123(2010e:42013)

[OS05] Kasso A. Okoudjou and Robert S. Strichartz. Weak uncertainty principles on fractals. J. Fourier Anal. Appl., 11(3):315-331, 2005. MR2167172(2006f:28011)

[Ped04] Steen Pedersen. On the dual spectral set conjecture. In Current trends in operator theory and its applications, volume 149 of Oper. Theory Adv. Appl., pages 487-491. Birkhäuser, Basel, 2004. MR2063764 (2005h:42016)

[Tao04] Terence Tao. Fuglede's conjecture is false in 5 and higher dimensions. Math. Res. Lett., 11(2-3):251-258, 2004. MR2067470(2005i:42037)

[Tho05] Klaus Thomsen. On the structure of beta shifts. In Algebraic and topological dynamics, volume 385 of Contemp. Math., pages 321-332. Amer. Math. Soc., Providence, RI, 2005. MR.2180243 (2006f:37012)

[YALC03] Zu-Guo Yu, Vo Anh, Ka-Sing Lau, and Ka-Hou Chu. The genomic tree of living organisms based on a fractal model. Phys. Lett. A, 317(3-4):293-302, 2003. MR.2018655

[ZHSS09] Haibiao Zheng, Yanren Hou, Feng Shi, and Lina Song. A finite element variational multiscale method for incompressible flows based on two local Gauss integrations. J. Comput. Phys., 228(16):5961-5977, 2009. MR2542923(2010h:76096)

University of Central Florida, Department of Mathematics, 4000 Central Florida Blvd., P.O. Box 161364, Orlando, Florida 32816-1364

E-mail address: ddutkay@mail.ucf.edu

University of Iowa, Department of Mathematics, 14 Maclean Hall, Iowa City, Iowa 52242-1419

E-mail address: jorgen@math.uiowa.edu 